\title{
Developments in continuous therapy and maintenance treatment approaches for patients with newly diagnosed multiple myeloma
}

Meletios A. Dimopoulos ${ }^{1}$, Andrzej J. Jakubowiak², Philip L. McCarthy ${ }^{3}$, Robert Z. Orlowskii, Michel Attal' ${ }^{4}$, Joan Bladé6, Hartmut Goldschmidt ${ }^{7}$, Katja C. Weisel ${ }^{8}$, Karthik Ramasamy ${ }^{9}$, Sonja Zweegman ${ }^{10}$, Andrew Spencer ${ }^{11}$, Jeffrey S. Y. Huang ${ }^{12}$, Jin Lu ${ }^{13}$, Kazutaka Sunami $\mathbb{B}^{14}$, Shinsuke lida ${ }^{15}$, Wee-Joo Chng ${ }^{16}$, Sarah A. Holstein ${ }^{17}$, Alberto Rocci ${ }^{18,19}$, Tomas Skacel ${ }^{20}$, Richard Labotka ${ }^{20}$, Antonio Palumbo ${ }^{20}$ and Kenneth C. Anderson ${ }^{21}$

\begin{abstract}
The evolving paradigm of continuous therapy and maintenance treatment approaches in multiple myeloma (MM) offers prolonged disease control and improved outcomes compared to traditional fixed-duration approaches. Potential benefits of long-term strategies include sustained control of disease symptoms, as well as continued cytoreduction and clonal control, leading to unmeasurable residual disease and the possibility of transforming MM into a chronic or functionally curable condition. "Continuous therapy" commonly refers to administering a doublet or triplet regimen until disease progression, whereas maintenance approaches typically involve single-agent or doublet treatment following more intensive prior therapy with autologous stem cell transplant (ASCT) or doublet, triplet, or even quadruplet induction therapy. However, the requirements for agents and regimens within these contexts are similar: treatments must be tolerable for a prolonged period of time, should not be associated with cumulative or chronic toxicity, should not adversely affect patients' quality of life, should ideally be convenient with a minimal treatment burden for patients, and should not impact the feasibility or efficacy of subsequent treatment at relapse. Multiple agents have been and are being investigated as long-term options in the treatment of newly diagnosed MM (NDMM), including the immunomodulatory drugs lenalidomide and thalidomide, the proteasome inhibitors bortezomib, carfilzomib, and ixazomib, and the monoclonal antibodies daratumumab, elotuzumab, and isatuximab. Here we review the latest results with long-term therapy approaches in three different settings in NDMM: (1) maintenance treatment post ASCT; (2) continuous frontline therapy in nontransplant patients; (3) maintenance treatment post-frontline therapy in the nontransplant setting. We also discuss evidence from key phase 3 trials. Our review demonstrates how the paradigm of long-term treatment is increasingly well-established across NDMM treatment settings, potentially resulting in further improvements in patient outcomes, and highlights key clinical issues that will need to be addressed in order to provide optimal benefit.
\end{abstract}

Correspondence: Meletios A. Dimopoulos (mdimop@med.uoa.gr) ${ }^{1}$ Hematology \& Medical Oncology, Department of Clinical Therapeutics, National and Kapodistrian University of Athens, School of Medicine, Athens, Greece

${ }^{2}$ University of Chicago Medical Center, Chicago, IL, USA

Full list of author information is available at the end of the article

\section{Introduction}

Outcomes in patients with multiple myeloma (MM) have improved substantially over the past two decades ${ }^{1}$. Ongoing increases in progression-free (PFS) and overall survival (OS) are being seen with novel regimens across treatment settings, associated with the evolving paradigm of long-term treatment approaches, including continuous therapy and maintenance, which can prolong disease 
control and improve PFS and sometimes OS compared to fixed-duration approaches ${ }^{2,3}$. Definitions of therapeutic approaches within this paradigm of long-term treatment are summarized in Table $1^{4,5}$.

This paradigm is being increasingly followed, with safety profiles of newer drugs improving long-term treatment feasibility vs. older agents ${ }^{2}$. Various long-term approaches in newly diagnosed MM (NDMM) are discussed within current guidelines and recommendations ${ }^{1,6-10}$. Consequently, and associated with benefits demonstrated in randomized clinical trials, long-term therapy is used extensively in routine clinical practice in some geographies. Maintenance was used in $81 \%$ of autologous stem cell transplant (ASCT) patients and $68 \%$ of nontransplant patients in 2017 US physician-reported data ${ }^{11}$. However, retrospective data on real-world practice patterns in Europe indicated only $12 \%$ of patients received maintenance as part of frontline treatment (acknowledging that this 2016 publication preceded the 2017 approval of lenalidomide in this setting) $)^{12}$.

We review the increasing importance of continuous therapy and maintenance in targeting the goal of improving outcomes and providing "functional cure" (i.e. long-term molecular remission ${ }^{1,13}$ ) in MM. We focus on long-term therapy in three settings: (1) maintenance treatment post ASCT; (2) continuous frontline therapy in nontransplant patients; (3) maintenance treatment post-frontline therapy in nontransplant patients. We highlight the latest evidence from phase 3 trials, plus emerging real-world data. We also consider practical requirements of long-term therapeutic approaches, including patient preferences and quality of life (QoL), tolerability and safety challenges, and pharmacoeconomics.

\section{Requirements/goals of long-term treatment}

Requirements for long-term treatment approaches are summarized in Table $2^{9,14-17}$. The key goals of long-term

Table 1 Definitions of therapeutic approaches within the paradigm of long-term treatment.

\begin{tabular}{|c|c|}
\hline Continuous therapy & Maintenance therapy \\
\hline $\begin{array}{l}\text {-Commonly refers to administering a regimen until } \\
\text { disease progression }\end{array}$ & -Commonly refers to treatment that differs from previous, more intensive therapy \\
\hline $\begin{array}{l}\text {-Typically a doublet or triplet, such as standard-of- } \\
\text { care } \mathrm{Rd}^{4}\end{array}$ & $\begin{array}{l}\text {-Typically single-agent or doublet therapy following ASCT, per the recent approval of single- } \\
\text { agent lenalidomide } \text {, or following doublet, triplet, or even quadruplet remission induction } \\
\text { therapy }\end{array}$ \\
\hline
\end{tabular}

ASCT autologous stem cell transplant, $R d$ lenalidomide-dexamethasone.

Table 2 Key requirements for long-term treatment approaches.

\begin{tabular}{|c|c|}
\hline Requirement & Specific needs for continuous therapy and maintenance treatment \\
\hline Efficacy/effectiveness & $\begin{array}{l}\text {-Agents/regimens must be active. } \\
\text {-Further long-term treatment options are needed that are efficacious across patient subgroups, including those with high- } \\
\text { risk disease }{ }^{17} \text {, for whom longer-term treatment is a particular requirement to achieve sustained disease control. } \\
\text {-Additional options are also needed that have demonstrated real-world feasibility and effectiveness, with no impact on } \\
\text { feasibility or efficacy of subsequent treatment at relapse. } \\
\text {-Given the heterogeneity of MM, long-term treatments incorporating multiple drugs with differing mechanisms of action } \\
\text { may be required for prolonged disease control in specific patient subgroups }{ }^{9} \text {. }\end{array}$ \\
\hline Tolerability/safety & $\begin{array}{l}\text {-Must be able to be tolerated for a prolonged period with little-to-no cumulative or chronic toxicity or substantive adverse } \\
\text { impact on patients' QoL. }\end{array}$ \\
\hline Minimal treatment burden & $\begin{array}{l}\text {-Minimal treatment burden through convenience of administration is important, highlighting the preference for all-oral } \\
\text { treatment options that avoid the patient and caregiver burden associated with repeat parenteral administration. } \\
\text {-Indeed, patient preference for all-oral vs. injectable proteasome inhibitor-based treatment has been reported in the } \\
\text { relapsed/refractory setting }{ }^{14} \text {. } \\
\text {-All-oral regimens have been shown to have lower economic burden of illness, less activity impairment, lower productivity } \\
\text { loss, and a trend towards greater convenience than injectable regimens in the frontline setting }{ }^{15,16} \text {. } \\
\text {-A minimal treatment and toxicity burden is also important in the context of patients potentially otherwise preferring a } \\
\text { treatment-free interval. }\end{array}$ \\
\hline
\end{tabular}

QoL quality of life. 
treatment are to prolong disease control and improve PFS and OS. Among the potential benefits are suppression of clonal evolution (recognizing that emergence of drugresistant clones is also a potential risk that could limit future treatment options) ${ }^{18}$; however, this hypothesis needs demonstrating in randomized controlled trials and is currently based on expert assumptions. Similarly, other potential benefits include sustained control of disease symptoms, immune modulation, and continued cytoreduction leading to unmeasurable residual diseaseoptimally, complete eradication of MM cells ${ }^{2}$. Deepening of response is an important goal, as deeper responses ${ }^{19}$ (and sustained deep response $\mathrm{e}^{20}$ ) are associated with improved outcomes. Converting patients to, and sustaining, minimal residual disease (MRD)-negative status represents a step towards "functional cure"13. Emerging data from continuous therapy and maintenance approaches have already demonstrated a positive impact on rates of MRD-negative disease status ${ }^{21-25}$.

\section{Post-ASCT maintenance therapy}

Key phase 3 data on agents investigated as post-ASCT maintenance therapy are summarized in Table 3.

\section{Immunomodulatory drugs}

Thalidomide maintenance has been studied in multiple phase 3 trials $^{26-29}$ and meta-analyses ${ }^{26,30}$, which generally showed a significant PFS benefit; a meta-analysis by the International Myeloma Working Group (IMWG) demonstrated a $35 \%$ reduction in risk of progression or death $^{30}$. However, less uniform findings have been reported regarding OS, with a significant benefit not found in the majority of individual studies but an overall significant improvement seen in the IMWG (hazard ratio (HR) 0.84$)^{30}$ and Myeloma IX-related (HR 0.75$)^{26}$ metaanalyses. Importantly, in some studies, limited durations of thalidomide maintenance and high discontinuation rates due to toxicity were reported ${ }^{26,27,29}$, as well as poorer survival following disease progression among patients exposed to thalidomide maintenance ${ }^{28}$, suggesting the selection of more resistant clones ${ }^{18}$. Thalidomide (vs. no maintenance) was associated with no PFS benefit and an adverse impact on OS in patients with high-risk cytogenetic abnormalities in the Myeloma IX trial (median OS 35 vs. 47 months $)^{26,31}$. Thalidomide is not approved as post-ASCT maintenance.

Multiple phase 3 studies of single-agent lenalidomide as post-ASCT maintenance have been reported (Table 3), with the meta-analysis ${ }^{32}$ of 1208 patients who received lenalidomide vs. placebo/no maintenance post ASCT in the CALGB $100104^{33-35}$, IFM2005-02 ${ }^{36}$, and GIMEMA RV-MM-PI-209 ${ }^{37}$ studies resulting in its approval in this setting (Table 3$)^{5,32}$. These studies showed substantial PFS benefit with lenalidomide vs. placebo/observation (HR
0.47-0.57), and significant OS improvements were reported in the CALGB and GIMEMA studies but not in IFM2005-02. In addition to early termination of maintenance due to a second primary malignancy (SPM) sig$\mathrm{nal}^{36}$, the fact that all patients in IFM2005-02 received lenalidomide consolidation post ASCT and that maintenance was not continued until progression may have contributed to the disparate OS findings. Importantly, in contrast to thalidomide, median OS post-relapse in CALGB 100104 appeared similar in the lenalidomide and placebo groups ${ }^{33,34}$. This is supported by recent reports showing lenalidomide maintenance resulting in prolonged time to disease progression on subsequent treatment (PFS2) and having no adverse impact on post-relapse survival $^{38}$, including in patients receiving subsequent immunomodulatory-drug-based therapies ${ }^{38,39}$.

Subgroup analyses from the meta-analysis of lenalidomide maintenance demonstrated a uniform PFS benefit (HRs 0.40-0.58) vs. placebo/no maintenance in patients regardless of age, disease stage, and post-ASCT response, although limited benefit was reported in some high-risk subgroups (renal impairment post ASCT, HR 0.79; elevated lactate dehydrogenase, HR 0.89; adverse-risk cytogenetics, HR 0.86$)^{32}$. However, incomplete data across studies precluded any definitive statement. OS findings were disparate, with no benefit seen in patients with stage III disease (HR 1.06), elevated lactate dehydrogenase (HR 1.17), or adverse-risk cytogenetics (HR 1.17). Additionally, OS benefit appeared more pronounced in patients achieving complete response (CR) or very good partial response (VGPR; HR 0.70) vs. <VGPR post ASCT (HR 0.88 ), and in patients who received lenalidomidecontaining ( $\mathrm{HR}$ 0.50) vs. non-lenalidomide (HR 0.82) induction $^{32}$. The benefits of lenalidomide maintenance vs. observation in terms of PFS, PFS2, and OS have also been reported from the transplant-eligible intensive pathway of the Myeloma XI trial, with significant improvements observed (Table 3$)^{25,40}$. This study had more patients with complete cytogenetic data and demonstrated improved PFS and OS with lenalidomide maintenance regardless of cytogenetic status, although absolute outcomes were poorer in high-risk patients ${ }^{25}$. Notably, median PFS improved by $\sim 16$ (ultra-high-risk patients) and $\sim 31$ months (high-risk patients) with lenalidomide vs. observation $^{25}$.

The value of lenalidomide alone or in combination as maintenance has been demonstrated in other key studies $(\text { Table 3) })^{41}$. In the IFM 2009 study, lenalidomide maintenance for 1 year following bortezomib-lenalidomidedexamethasone (VRd) induction plus ASCT vs. prolonged VRd increased the $\geq$ VGPR rate $(78 \%$ vs. $69 \%$ to $85 \%$ vs. $76 \%$, respectively ${ }^{42}$. Similarly, an ongoing phase 2 study of lenalidomide-elotuzumab as post-ASCT maintenance showed response improvements in $33 \%$ of patients, with 


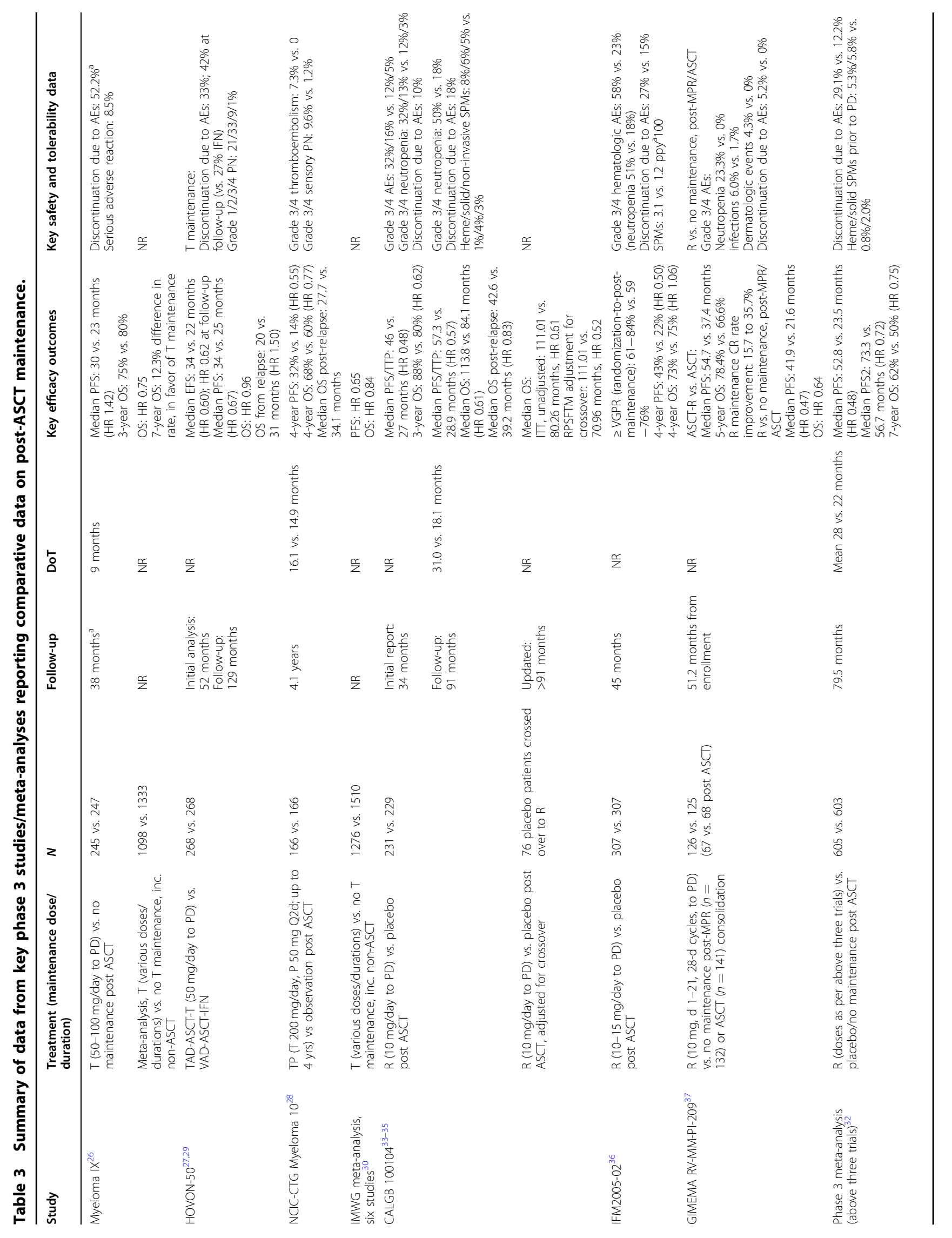




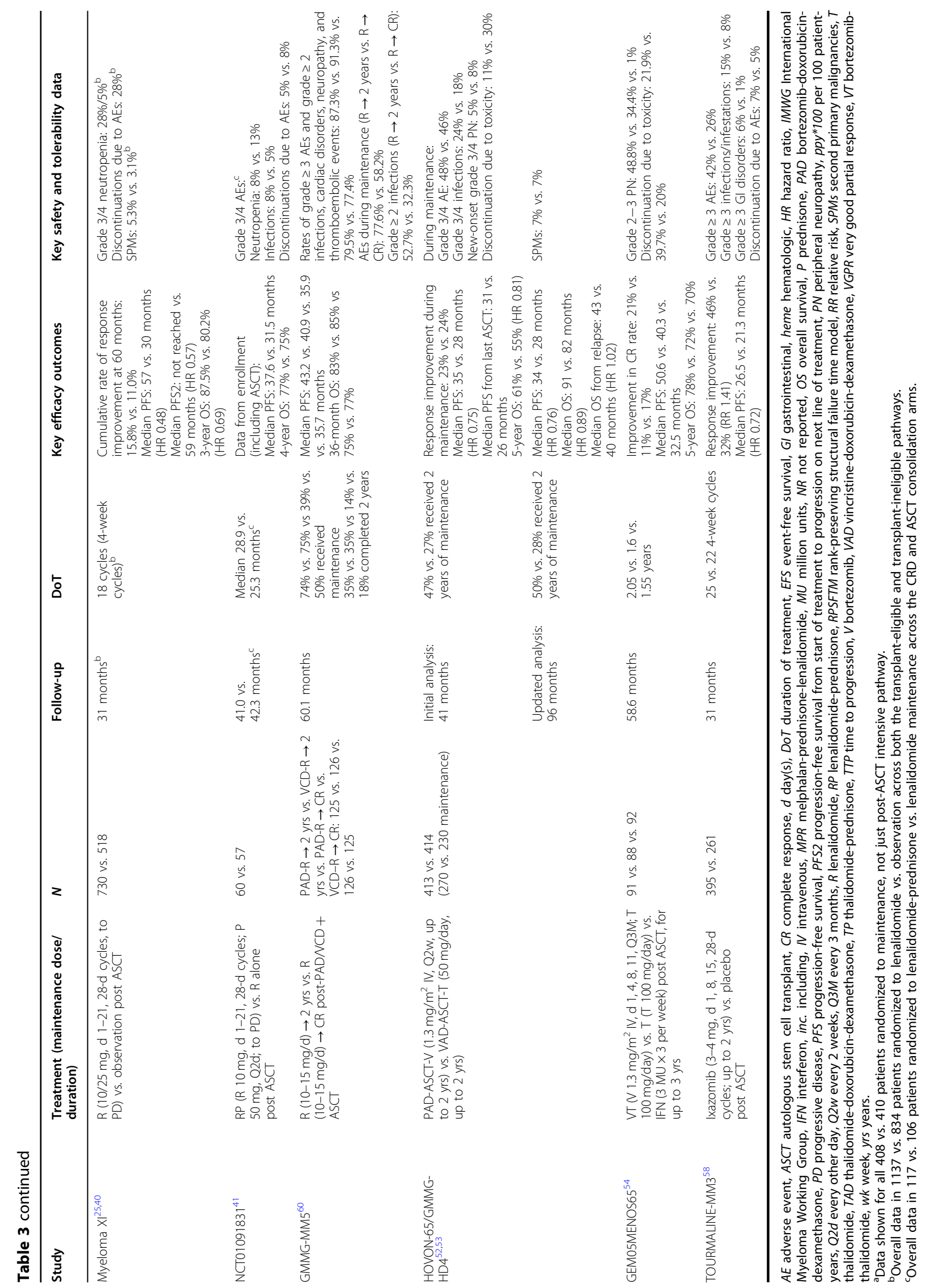


$20 \%$ converting to $\mathrm{CR}^{43}$. Additionally, while some patients $\left(4-7 \%^{44,45}\right)$ may convert to MRD-negative status post ASCT without requiring maintenance, analyses of studies employing lenalidomide maintenance, including Myeloma XI, EMN02/HO95, and RV-MM-EMN-441, have demonstrated substantially higher rates of conversion from MRD-positive to MRD-negative status of $\sim 27-48 \%{ }^{24,44,46}$.

Importantly, the value of lenalidomide maintenance is being demonstrated in the real-world setting. Reports from the Connect ${ }^{\circledR}$ MM registry, a US noninterventional, prospective registry incorporating $>3000$ NDMM patients from 250 academic-, government-, and community-based centers, have highlighted that lenalidomide maintenance post ASCT results in improved PFS (median 54.5 vs. 30.4 months, HR 0.58 ) and OS (3-year rate: $85 \%$ vs. $70 \%$; HR 0.45$)$ vs. no maintenance ${ }^{47,48}$, and that maintenance has no adverse impact on $\mathrm{QoL}^{49}$. Real-world analyses from the Mayo Clinic (lenalidomide vs. no maintenance: median PFS 37 vs. 28 months, HR 0.48) ${ }^{50}$ and the Princess Margaret Cancer Centre in Toronto (median PFS 41.7 months with lenalidomide maintenance) ${ }^{51}$ have reflected efficacy findings from clinical trials, but at the cost of some tolerability, with $17 \%^{50}$ and $13 \%$ of patients $^{51}$, respectively, discontinuing due to toxicity, and $70 \%$ requiring dose reductions in the Toronto study ${ }^{51}$.

\section{Proteasome inhibitors}

Bortezomib-based maintenance post ASCT has been evaluated in two key phase 3 studies. In the HOVON-65/ GMMG-HD4 study ${ }^{52,53}$, single-agent bortezomib maintenance for 2 years following bortezomib-based induction and ASCT contributed to improved response rates and outcomes vs. single-agent thalidomide maintenance for 2 years following vincristine-doxorubicin-dexamethasone induction and ASCT (Table 3); however, the isolated benefit of bortezomib vs. thalidomide maintenance was not entirely clear as patients were not re-randomized post ASCT. Bortezomib maintenance was better tolerated, with $11 \%$ of patients discontinuing due to toxicity vs. $30 \%$ with thalidomide; however, $13 \%$ of patients discontinued prior to bortezomib maintenance due to toxicity, primarily polyneuropathy. In the GEM05MENOS65 study ${ }^{54}$, patients were randomized to one of three induction regimens and then re-randomized to compare post-ASCT maintenance for $\leq 3$ years with bortezomib-thalidomide (VT), thalidomide alone, or interferon. VT maintenance resulted in the greatest improvement in CR rate and the longest PFS, but OS was similar in all three maintenance arms (Table 3).

In HOVON-65/GMMG-HD4, long-term bortezomibbased treatment appeared to abrogate the poor prognostic impact of del(17p), with 8 -year OS rates of $52 \%$ vs. $54 \%$ in patients with and without this cytogenetic abnormality, which was not a stratification factor ${ }^{52}$. However, the poor prognostic impact of other high-risk cytogenetic abnormalities- $t(4 ; 14)$ and gain $1 \mathrm{q} 21$-was not overcome $^{52}$. A recent analysis proposed that this was associated with additional subclonal heterogeneity ${ }^{55}$, suggesting the need for combination continuous therapy strategies in such high-risk patients. One single-center analysis has suggested that VRd consolidation and maintenance post ASCT may be promising for patients with high-risk disease (del17p, del1p, $\mathrm{t}(4 ; 14), \mathrm{t}(14 ; 16)$; $96 \% \geq$ VGPR, median PFS 32 months, 3-year OS 93\%) ${ }^{56}$. Additionally, a phase 2 study has evaluated intensive bortezomib-based triplet therapy as post-ASCT maintenance in elderly patients, including $40 \%$ with high-risk disease, with promising early findings ${ }^{57}$. Notably, bortezomib maintenance post ASCT has demonstrated a PFS benefit (median 28 vs. 16 months) in high-risk patients in the real-world setting, in a retrospective, single-center analysis at Mayo Clinic ${ }^{50}$. However, the role of bortezomib-based therapy solely as maintenance cannot be extrapolated from these studies, in which patients may have also received bortezomib-based induction.

The recent phase 3 TOURMALINE-MM3 study showed, for the first time, the benefit of a proteasome inhibitor vs. placebo as post-ASCT maintenance, with the oral proteasome inhibitor ixazomib demonstrating a statistically significant PFS benefit (median 26.5 vs. 21.3 months, HR 0.72; HR 0.62 in 115 patients with highrisk cytogenetics) and a significantly greater rate of response improvement vs. placebo (Table 3$)^{58}$. Ixazomib maintenance was planned for up to 2 years; $50 \%$ of patients completed the maximum duration, with $7 \%$ discontinuing due to toxicity and $36 \%$ due to progressive disease. With a median follow-up of 31 months, PFS2 and OS data were not mature, with follow-up ongoing. Additional studies are evaluating ixazomib maintenance in combination with existing agents. For example, a phase 2 study has demonstrated the feasibility and activity of long-term ixazomib-lenalidomide therapy as post-ASCT maintenance ${ }^{59}$. The doublet improved responses in $45 \%$ of patients, and median PFS has not been reached after a median follow-up of $>3$ years ${ }^{59}$. Only $6 \%$ of patients discontinued ixazomib due to toxicity ${ }^{59}$, providing further evidence for its feasibility as a component of long-term treatment approaches.

\section{Optimal duration of post-ASCT maintenance}

An outstanding question in post-ASCT maintenance is regarding optimal duration of treatment. The studies included in a meta-analysis of lenalidomide maintenance in key phase 3 trials (in which the mean treatment duration was 28 months) were all of the treat-toprogression approach ${ }^{32,34,36,37}$. However, there are other trials that use a fixed-duration approach for 1-2 
years $^{42,60}$. Comparative studies of these approaches, and of fixed-duration maintenance vs. placebo, are not available. However, it is important to balance potential benefits and risks. Some patients may derive an optimal benefit/ risk balance from shorter-term/fixed-duration lenalidomide maintenance (similar to findings with thalidomide maintenance of relatively limited duration ${ }^{26,28,30}$ ), whereas in other settings a longer treatment duration may be warranted. For example, in the phase 3 GMMG-MM5 trial (Table 3), patients received lenalidomide maintenance post ASCT for either 2 years or until they achieved $C^{60}$. No significant difference in PFS was seen between groups but 3-year OS rates were significantly higher in the 2-year treatment group. However, this was accompanied by a significant increase in toxicity. Nevertheless, results suggest that lenalidomide maintenance beyond $\mathrm{CR}$ achievement offers improved outcomes ${ }^{60}$. Similar conclusions have been reported from a pooled analysis in the post-ASCT and nontransplant settings, which demonstrated prolonged survival with maintenance vs. no maintenance in patients achieving a CR postinduction/consolidation, thereby indicating the importance of continuing treatment in these patients ${ }^{61}$. To date, fixed-duration approaches have been used in studies of proteasome inhibitor-based maintenance ${ }^{53,54,58}$, leaving the question of whether longer treatment might have further improved outcomes.

In this context, a follow-up question might be: at what depth of response might maintenance be stopped without affecting outcomes? The potential utility of MRD status for determining use and/or duration of maintenance therapy has been reviewed previously and potential study designs have been suggested to evaluate whether MRDnegative patients require ongoing therapy ${ }^{13}$. Data from Myeloma XI showed a PFS advantage with lenalidomide maintenance regardless of MRD status and demonstrated an increased rate of conversion from MRD-positive to MRD-negative status with lenalidomide (32\%) vs. observation $(4 \%)^{44}$. Preliminary data from another study suggest that MRD-negative status conferred high PFS values regardless of lenalidomide maintenance use (2-year PFS $88 \%$ vs. $74 \%$ ), whereas in MRD-positive patients lenalidomide vs. no maintenance resulted in a significantly higher 2 -year PFS rate $(94 \% \text { vs. } 45 \%)^{62}$. In TOURMALINE-MM3, median PFS with ixazomib vs. placebo maintenance was 38.6 vs. 32.5 months (HR 0.61) in patients who were MRD-negative at study entry and 23.1 vs. 18.5 months (HR 0.70) in MRD-positive patients ${ }^{58}$. Further investigation is warranted, utilizing increasingly sensitive MRD assessment techniques, to determine whether MRD status can guide duration of post-ASCT maintenance-and of continuous therapy more broadly ${ }^{13}$-with an increasing number of trials demonstrating high rates of MRD-negativity (e.g. with lenalidomide maintenance $e^{24,44,46}$ ) and incorporating MRD status as a clinical and regulatory endpoint (Table 4).

\section{Ongoing randomized comparative studies}

There are several ongoing randomized comparative studies yet to report data that are addressing the specific impact of newer agents within the post-ASCT maintenance setting (Table 4). The GEM2014MAIN study is evaluating addition of ixazomib to lenalidomidedexamethasone $(\mathrm{Rd})$ as maintenance, while the phase 3 ATLAS and FORTE studies are assessing carfilzomib-R(d) vs. lenalidomide in this setting, with data from the induction/consolidation phase of FORTE having already been reported ${ }^{63}$. The Cassiopeia study includes postASCT randomization to daratumumab maintenance vs. observation, the EMN18 study is evaluating addition of daratumumab to ixazomib maintenance, while daratumumab, elotuzumab, and isatuximab are being studied in combination with lenalidomide as post-ASCT maintenance in studies by the SouthWest Oncology Group (SWOG) and the German-speaking Multicenter Myeloma Group.

\section{Continuous frontline therapy in the nontransplant setting \\ Current treatment approaches}

Since initial publication of the phase 3 FIRST trial ${ }^{64}$, continuous $\mathrm{Rd}$ has emerged as a standard-of-care frontline therapy, with other continuous treatment regimens building upon this doublet. FIRST evaluated the outcome benefits of continuous Rd vs. fixed-duration $\mathrm{Rd}$ for 18 cycles (Rd18) vs. fixed-duration melphalan-prednisonethalidomide $(\mathrm{MPT})^{64}$. At the initial analysis, PFS was improved with continuous $\mathrm{Rd}$ vs. Rd18 and vs. MPT, response rates were higher with continuous $\mathrm{Rd}$ and $\mathrm{Rd} 18$ vs. MPT, and OS rates were higher with continuous Rd vs. MPT (Table 5$)^{64}$. The subsequent final analysis confirmed these findings-the 4-year PFS rate with continuous $\mathrm{Rd}$ was more than double those with Rd18 and MPT; furthermore, there was a significant OS benefit with continuous Rd vs. MPT, although OS was similar with continuous $\mathrm{Rd}$ and $\mathrm{Rd} 18^{4}$.

The benefit of continuous Rd vs. MPT has been demonstrated in multiple patient subgroups ${ }^{4}$, including those achieving $C R, \geq V G P R$, and $\geq \mathrm{PR}^{65}$, those with no, mild, or moderate renal impairment ${ }^{66}$, and those aged $\leq 75$ or $>75$ years $^{4}$. However, recently reported data from the RV-MM-PI-0752 study comparing continuous Rd with $\mathrm{Rd}$ followed by lenalidomide maintenance (Rd-R) in elderly and intermediate-fit NDMM patients showed no significant differences in efficacy between regimens but lower rates of adverse events (AEs) and dose reductions in the Rd-R arm (Table 5) ${ }^{67}$. These findings suggest that continuous Rd may not represent an optimal approach for 
Table 4 Ongoing phase 3 and randomized phase 2 comparative studies of continuous therapy and maintenance treatment approaches that have not yet reported data at the time of publication (ClinicalTrials.gov, April 26, 2019).

\begin{tabular}{|c|c|c|c|c|c|c|}
\hline Study & NCT number & Phase & $\begin{array}{l}\text { Maintenance/continuous } \\
\text { treatment regimens }\end{array}$ & $N$ & Primary endpoint & $\begin{array}{l}\text { Estimated } 1^{\circ} \\
\text { completion date }\end{array}$ \\
\hline \multicolumn{7}{|c|}{ Post-ASCT maintenance therapy } \\
\hline GEM2014MAIN & NCT02406144 & 3 & Ixazomib-Rd vs. Rd & 316 & PFS & Not known \\
\hline MMRC & NCT02253316 & 2 & Ixazomib vs. R & 240 & MRD & November 2019 \\
\hline NCl-2015-00138 & NCT02389517 & 2 & Ixazomib-Rd vs. R & 86 & MRD & March 2020 \\
\hline ATLAS & NCT02659293 & 3 & Carfilzomib-Rd vs. R & 180 & PFS & March 2019 \\
\hline FORTE & NCT02203643 & 2 & Carfilzomib-R vs. $R$ & 477 & $\begin{array}{l}\geq V G P R \text { rate post- } \\
\text { induction }\end{array}$ & October $2016^{a}$ \\
\hline Cassiopeia & NCT02541383 & 3 & Daratumumab vs. observation & 1085 & PFS & August 2022 \\
\hline $\mathrm{EMN} 18^{\mathrm{b}}$ & NCT03896737 & 2 & $\begin{array}{l}\text { Daratumumab-ixazomib vs. } \\
\text { ixazomib }\end{array}$ & 400 & $\begin{array}{l}\text { MRD-neg rate; } \\
\text { 2-year PFS }\end{array}$ & February 2022 \\
\hline AURIGA/MMY3021 & NCT03901963 & 3 & Daratumumab-R vs. $R$ & 214 & $\begin{array}{l}\text { MRD-neg rate at } \\
12 \text { months }\end{array}$ & May 2021 \\
\hline GRIFFIN/MMY2004 & NCT02874742 & 2 & Daratumumab-R vs. $R$ & 222 & $\begin{array}{l}\text { SCR rate post- } \\
\text { consolidation }\end{array}$ & January 2019 \\
\hline DraMMatic $^{c}$ & $\begin{array}{l}\text { SWOG1803/BMT } \\
\text { CTN } 1706\end{array}$ & 3 & Daratumumab-R vs. $R$ & Not known & Not known & Not known \\
\hline GMMG-HD6 & NCT02495922 & 3 & Elotuzumab-R vs. R & 564 & PFS & June 2020 \\
\hline GMMG-HD7 & NCT03617731 & 3 & Isatuximab-R vs. $R$ & 662 & PFS & May 2025 \\
\hline \multicolumn{7}{|c|}{ Continuous frontline therapy, non-ASCT setting } \\
\hline TOURMALINE-MM2 & NCT01850524 & 3 & Ixazomib-Rd vs. placebo-Rd & 701 & PFS & February 2018 \\
\hline COBRA & NCT03729804 & 3 & Carfilzomib-Rd vs. VRd & 250 & PFS & December 2021 \\
\hline GEM2017FIT & NCT03742297 & 3 & $\begin{array}{l}\text { Daratumumab + carfilzomib-Rd vs. } \\
\text { carfilzomib-Rd vs. VMP-Rd }\end{array}$ & 300 & $C R$ rate & October 2020 \\
\hline Perseus & NCT03710603 & 3 & $\begin{array}{l}\text { Daratumumab- } \\
\text { VRd-daratumumab-R vs. VRd-R }\end{array}$ & 690 & PFS & May 2029 \\
\hline MMY3019 & NCT03652064 & 3 & $\begin{array}{l}\text { Daratumumab- } \\
\text { VRd-daratumumab-Rd vs. VRd-Rd }\end{array}$ & 360 & MRD-neg rate & March 2024 \\
\hline ELOQUENT-1 & NCT01335399 & 3 & Elotuzumab-Rd vs. Rd & 750 & PFS & May 2019 \\
\hline SWOG S1211 & NCT01668719 & 2 & Elotuzumab-VRd vs. VRd & 122 & PFS & May 2019 \\
\hline IMROZ & NCT03319667 & 3 & $\begin{array}{l}\text { Isatuximab-VRd-isatuximab-Rd vs. } \\
\text { VRd-Rd }\end{array}$ & 440 & PFS & December 2022 \\
\hline \multicolumn{7}{|c|}{ Post-induction maintenance therapy, non-ASCT setting } \\
\hline $\begin{array}{l}\text { TOURMALINE-MM4 + } \\
\text { China continuation }\end{array}$ & $\begin{array}{l}\text { NCT02312258 } \\
\text { NCT03748953 }\end{array}$ & 3 & Ixazomib vs. placebo & $\begin{array}{l}706 \\
105\end{array}$ & PFS & $\begin{array}{l}\text { August } 2019 \\
\text { September } 2024\end{array}$ \\
\hline Myeloma XIV (FiTNEss) & NCT03720041 & 3 & $\begin{array}{l}\text { Ixazomib-R vs. placebo-R (post- } \\
\text { ixazomib-Rd) }\end{array}$ & 740 & PFS & December 2024 \\
\hline X16108 & NCT03733691 & 2 & Ixazomib-R vs. ixazomib & 52 & PFS, AEs & December 2023 \\
\hline AGMT_MM-2 & NCT02891811 & 2 & Carfilzomib vs. observation & 146 & Post-induction ORR & September 2023 \\
\hline
\end{tabular}

AEs adverse events, ASCT autologous stem cell transplant, $C R$ complete response, MRD-neg negative for minimal residual disease, ORR overall response rate, $P F S$ progression-free survival, $R$ lenalidomide, $R d$ lenalidomide-dexamethasone, VMP bortezomib-melphalan-prednisone, VRd bortezomib-lenalidomide-dexamethasone. ${ }^{a}$ Data reported from induction/consolidation phase ${ }^{63}$; data not yet reported from the randomized maintenance phase of the study.

${ }^{b}$ Includes information from https://www.myeloma-europe.org/trials/emn-18/.

'Information from https://www.swog.org/clinical-trials/s1803.

these patients due to tolerability issues associated with long-term use of both agents, and a frailty-adjusted approach to long-term treatment of NDMM is needed.

In FIRST, continuous Rd did not appear to offer consistent benefit vs. MPT according to cytogenetic risk status ${ }^{4}$. Among patients with standard-risk cytogenetic abnormalities, there was a significant PFS (HR 0.66) and OS (HR 0.69) benefit with continuous Rd, but patients with high-risk cytogenetics had similar outcomes with each therapy (PFS HR 1.27, OS HR 0.92) ${ }^{4}$. The authors suggested that triplet regimens built upon the continuous $\mathrm{Rd}$ backbone may be required in high-risk patients.
The phase 3 MAIA study of daratumumab-Rd vs. Rd to progression recently demonstrated the feasibility and activity of such a continuous triplet therapy (Table 5$)^{68}$. In the initial analysis, daratumumab-Rd resulted in a significant $44 \%$ reduction in risk of progression or death, and response rates were significantly higher. Median OS was not reached in either arm; additional follow-up is required to evaluate long-term tolerability and efficacy.

The SWOG S0777 study also demonstrated the benefit of a triplet regimen (VRd) vs. Rd in the NDMM setting ${ }^{69,70}$; however, unlike in MAIA, VRd was administered for only eight cycles before patients discontinued 


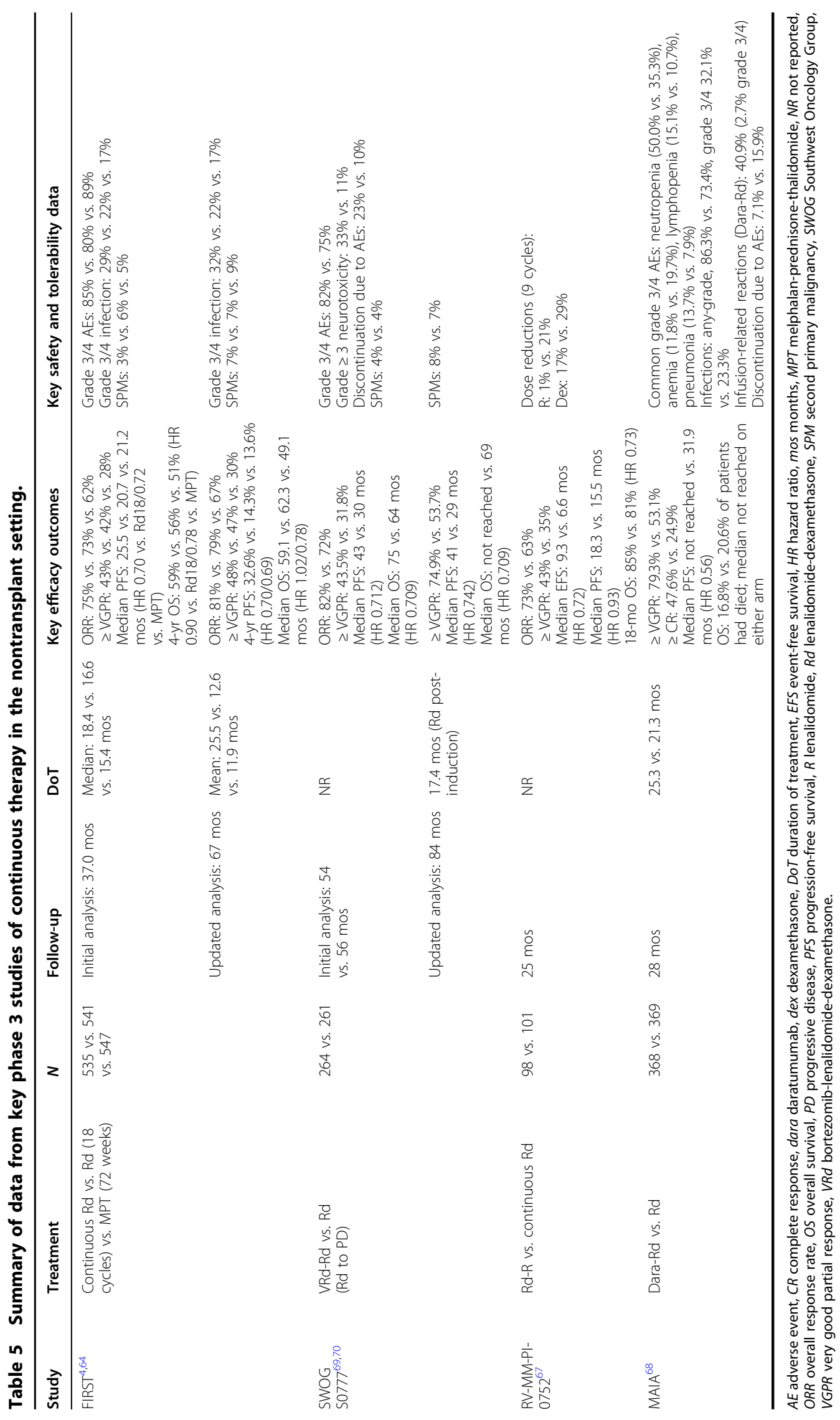


bortezomib and continued Rd until progression. Nevertheless, the approach resulted in significant improvements in PFS and OS at the initial analysis that were maintained at an updated analysis after a median follow-up of 7 years (Table 5). Of note, median PFS was 38 vs. 16 months in the subgroup of 44 patients with high-risk disease by FISH, although this difference was not significant. The triplet appeared less tolerable than $\mathrm{Rd}$, with substantially higher rates of grade $\geq 3$ neurotoxicity and discontinuations due to AEs associated with the eight cycles of bortezomib therapy ${ }^{69}$; this could have been due to the use of intravenous instead of subcutaneous bortezomib, which has since become standard. Further studies are necessary to determine whether prolonged proteasome inhibitor therapy in addition to $\mathrm{Rd}$ could further improve outcomes.

A network meta-analysis in the setting of nontransplant NDMM has reinforced the findings from individual studies described above, albeit recent findings from MAIA were not included ${ }^{71}$. The analysis included studies of continuous $\mathrm{Rd}$ and VRd, and approaches utilizing a finite treatment duration or a post-induction maintenance approach (see next section). It found that, among approved treatment options, continuous $\mathrm{Rd}$ offered superior PFS and OS, and that among emerging treatment options only VRd resulted in significant improvements vs. continuous $\mathrm{Rd}^{71}$.

\section{Ongoing randomized comparative studies}

Several randomized comparative studies of continuous triplet and quadruplet therapies are ongoing (Table 4). The benefit of adding ixazomib or elotuzumab to $\mathrm{Rd}$ is being investigated in the TOURMALINE-MM2 and ELOQUENT-1 studies, respectively; carfilzomib-Rd is being compared with VRd in the head-to-head COBRA study, and daratumumab and isatuximab are being investigated in combination with a VRd/Rd or carfilzomib-Rd backbone. These quadruplet regimens are being investigated primarily in younger and/or fitter patients, and, if tolerable, may offer substantial rates of sustained MRD-negativity and prolonged outcomes.

\section{Maintenance therapy post-induction in the nontransplant setting}

In addition to continuous Rd being used alone and as a backbone for other long-term treatment, lenalidomide has been investigated as post-induction maintenance therapy, most commonly in "continuous lenalidomide" schemas involving lenalidomide-based induction followed by single-agent lenalidomide maintenance. Other agents, including thalidomide, bortezomib, ixazomib, and daratumumab, have been similarly studied in this setting (Table 6).
The efficacy of lenalidomide maintenance vs. observation post-lenalidomide/thalidomide-based induction in the nontransplant pathway of Myeloma XI has been reported recently ${ }^{25,40}$; results demonstrated a significant improvement in PFS and PFS2, but no OS benefit was seen (Table 6$)^{40}$. Notably, the PFS benefit of lenalidomide maintenance was seen regardless of cytogenetic risk. Lenalidomide also improved depth of response in approximately one-fifth of patients ${ }^{25}$. Similarly, the MM$015^{72}$ and GIMEMA-RV-MM-PI-209 ${ }^{37}$ studies have investigated the value of lenalidomide maintenance vs. placebo/observation following non-ASCT induction with melphalan-prednisone-lenalidomide (MPR) (Table 6). The "continuous lenalidomide" MPR-R schema resulted in significantly prolonged PFS compared to MPR induction alone in both studies; however, while a higher 5-year OS rate was seen with MPR-R in the GIMEMA RV-MMPI-209 study, no significant OS benefit was reported for MPR-R vs. MPR-placebo or MP-placebo in MM-015. Of note, in MM-015 the PFS benefit with MPR-R was only seen in patients aged 65-75 years (median 31 vs. 15 months in MPR-placebo, vs. 12 months in the MP control arm) and not in patients aged $>75$ years, possibly associated with poorer tolerability of the triplet in this population; specifically, rates of grade 4 hematologic toxicities and discontinuations due to AEs were markedly higher with MPR vs. $\mathrm{MP}^{72}$. However, in a landmark analysis to isolate the activity of lenalidomide maintenance, there was a clear PFS benefit with continued lenalidomide therapy vs. placebo, both overall and regardless of age ${ }^{72}$. These findings further reinforce the importance of treatment tolerability with regards to overall feasibility of continuous therapy approaches, particularly for elderly and/or frail populations.

Additional phase 3 studies of continuous therapy approaches have compared MPR-R vs. MPT-T (Table $6)^{73,74}$. Due to their designs, these studies were not able to demonstrate the isolated benefit of post-induction maintenance with an immunomodulatory drug. Data from the HOVON87/NMSG18 and E1A06 trials showed no significant efficacy differences between the two regimens; however, there was greater toxicity in the thalidomide $\mathrm{arms}^{73}$. Other phase 3 studies, including EMN01 ${ }^{75}$ and a European-Australian study ${ }^{41}$, have compared lenalidomideprednisone to lenalidomide as maintenance following lenalidomide-based induction. Data specific to the impact of post-induction maintenance therapy have not been reported, but the European-Australian study showed similar PFS and OS from the start of induction with the two regimens, and an overall analysis of maintenance patients, including those receiving post-ASCT maintenance, showed similar rates of toxicity between lenalidomide-prednisone and lenalidomide ${ }^{41}$. The authors concluded that the 


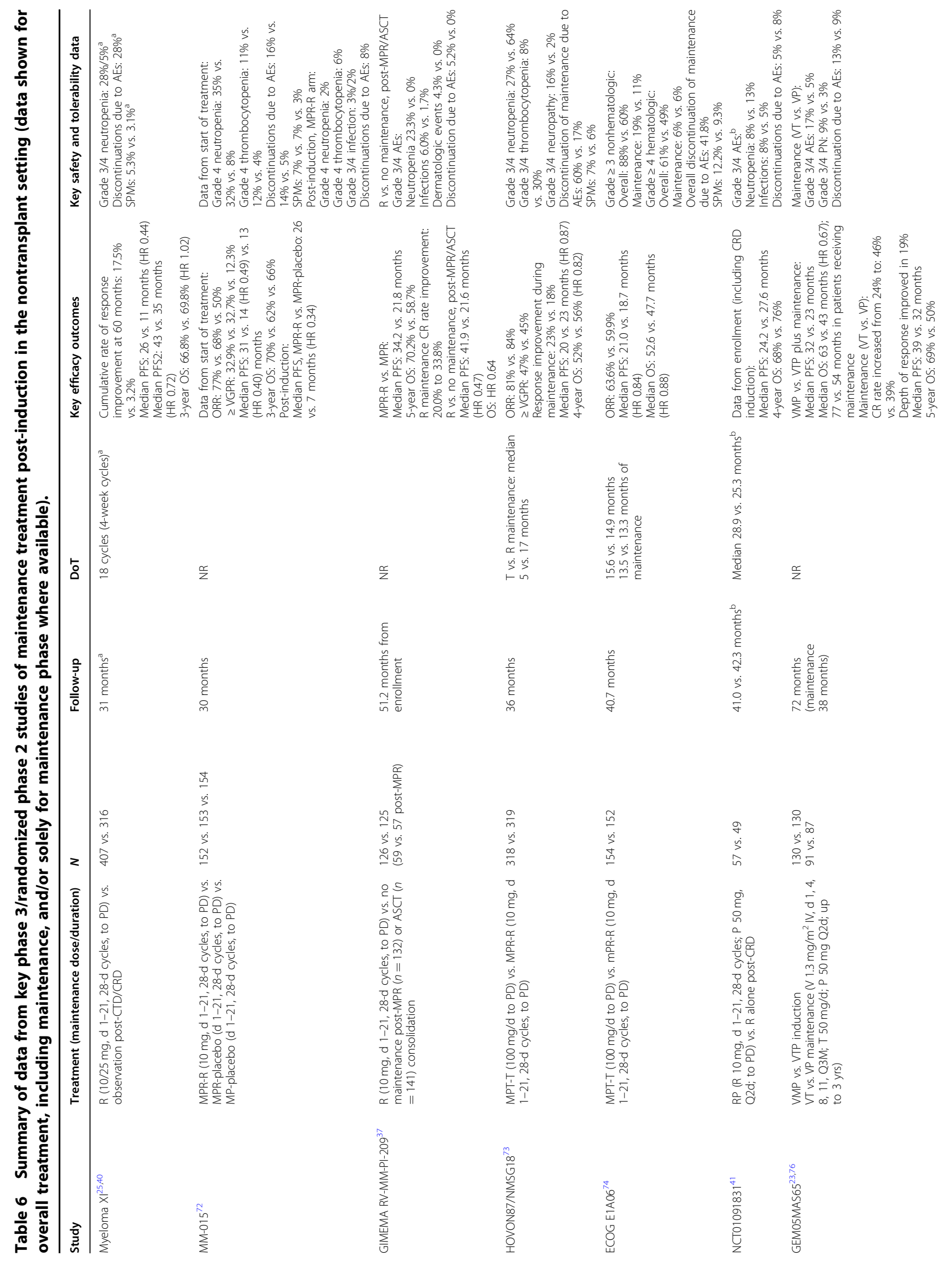


Dimopoulos et al. Blood Cancer Journal (2020)10:17

Page 12 of 19

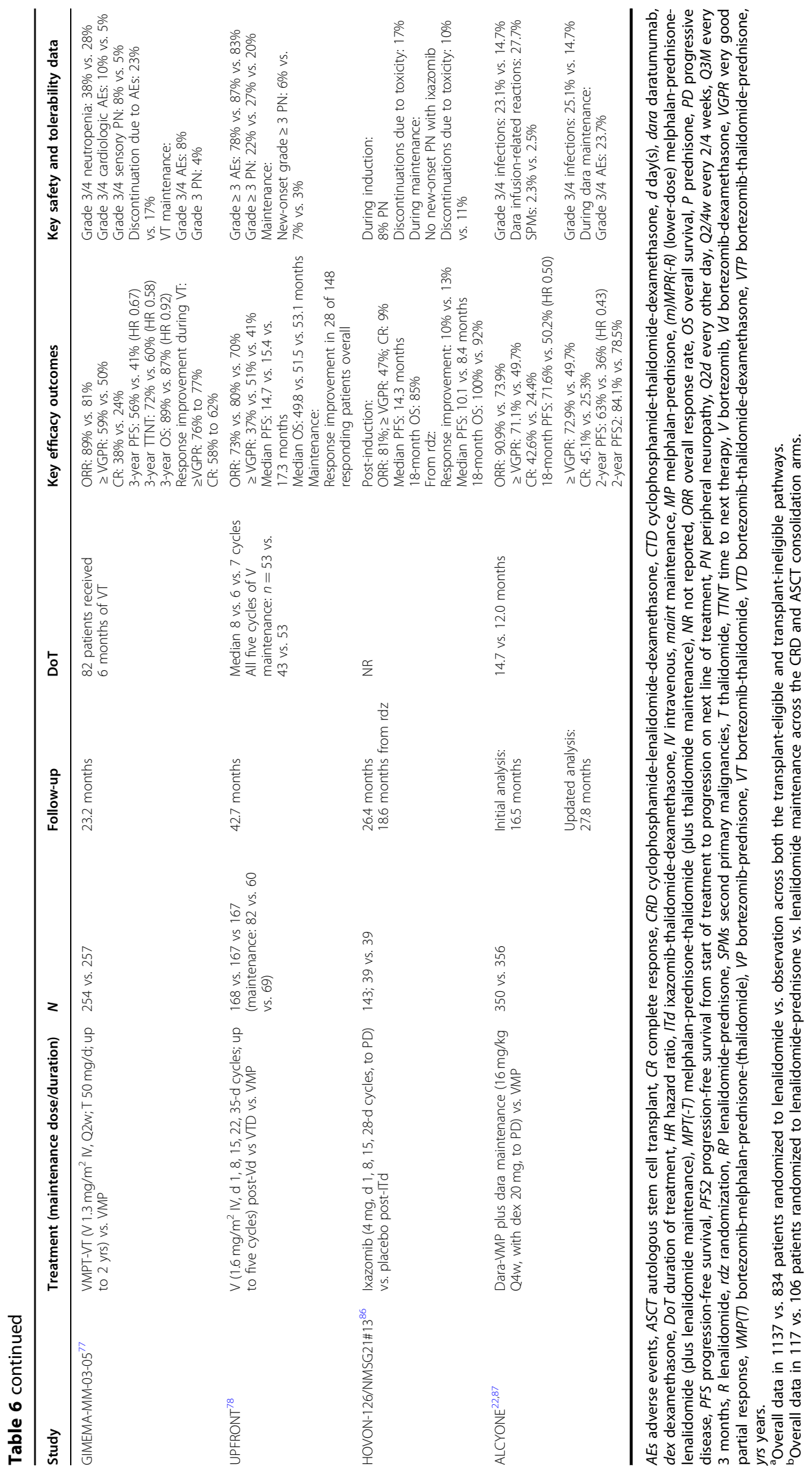

Blood Cancer Journal 
advantage of adding steroids to immunomodulatory drugs during maintenance is unclear.

There have been no randomized phase 3 studies demonstrating the specific benefit of proteasome inhibitorbased or monoclonal antibody-based maintenance within this setting; however, proteasome inhibitor maintenance has been shown to be feasible and active following proteasome inhibitor-based induction. Bortezomib-based maintenance following bortezomib-based induction resulted in substantial increases in CR rate and contributed to lengthy outcomes in the GEM05MAS65 study ${ }^{23,76}$, and contributed to improved PFS vs. a no-maintenance approach in the GIMEMA-MM-03-05 study (Table 6) ${ }^{77}$. In the phase $3 \mathrm{~B}$ community-based UPFRONT study, fixedduration single-agent bortezomib maintenance following bortezomib-based induction improved response depth in approximately $16 \%$ of patients, with limited new-onset toxicity $^{78}$. A phase $1 / 2$ study has shown the feasibility and activity of carfilzomib maintenance following weekly carfilzomib-cyclophosphamide-dexamethasone induction, with response improvements again being reported ${ }^{79}$.

Similarly, single-agent ixazomib maintenance has been utilized following ixazomib-based induction in four phase $1 / 2$ studies $^{45,80-83}$; a pooled analysis of maintenance patients from these studies reported deepening responses in $23 \%$ of patients, as well as a median PFS of 21.4 months and a 3-year OS of $82 \%$ from the start of maintenance, with limited new-onset $\mathrm{AEs}^{84}$. Ixazomib-daratumumabdexamethasone followed by ixazomib maintenance is being evaluated in unfit and frail patients in the phase 2 HOVON-143 study, which has reported promising safety and response data for the induction phase ${ }^{85}$. However, in the randomized phase 2 HOVON-126/NMSG21\#13 trial, in which patients received ixazomib-thalidomidedexamethasone induction and were then randomized to ixazomib or placebo maintenance ${ }^{86}$, preliminary data showed no response or PFS benefit with ixazomib maintenance to date, although ixazomib did not result in additional toxicity compared to placebo (Table 6). The ongoing phase 3 TOURMALINE-MM4 study will provide more comprehensive information on the use of single-agent ixazomib maintenance in the post-induction setting (Table 4).

The ALCYONE study has recently reported a substantial PFS benefit of continuous daratumumab treatment in the nontransplant setting, utilizing daratumumab-VMP plus daratumumab maintenance vs. VMP alone (Table 6) ${ }^{22,87}$. This long-term treatment approach has demonstrated PFS and PFS2 improvements vs. VMP, although OS data are not yet mature. Daratumumab maintenance was associated with a limited rate of grade $3 / 4 \mathrm{AEs}^{87}$. Network metaanalyses in the non-ASCT setting ${ }^{71,88}$ and matched-pair patient analyses ${ }^{89}$ support the efficacy of daratumumabVMP plus daratumumab maintenance vs. other treatment approaches. However, updated PFS curves suggest an increased rate of PFS events after 12 months in both $\mathrm{arms}^{87}$, following completion of the VMP component of therapy, suggesting the potential value of continuing proteasome inhibitor therapy with daratumumab maintenance.

\section{Safety and tolerability of long-term treatment approaches}

Toxicity and treatment burden may limit treatment duration and drive patients' preference for a treatment-free interval. Therefore, tolerability, limited treatment burden, absence of cumulative or chronic toxicity, and no adverse impact on QoL are important aspects for agents intended for continuous therapy or maintenance vs. fixed duration. The preceding sections have highlighted the substantial efficacy demonstrated by multiple agents in different treatment settings, but data from key studies (Tables 3, 5, 6) also show that regimens may be associated with safety and tolerability concerns that require consideration when selecting a long-term treatment approach. A recent retrospective study indicated no impact on PFS or OS of maintenance therapy with lenalidomide vs. bortezomib, the authors suggesting that side-effect profile and anticipated tolerability might be more valuable in guiding treatment choices $^{90}$. However, patients were heterogeneously treated and for varying degrees of time beyond 2 years of maintenance; thus, findings of this retrospective analysis should be interpreted with caution.

The findings from phase 3 studies reviewed herein have highlighted the differential feasibility of some agents as long-term therapy, due to tolerability limiting treatment durations and increasing rates of discontinuation due to AEs (Table 3); indeed, for some agents/regimens, based on the benefit/risk balance, a fixed-duration approach may be more appropriate for some patients. Long-term therapy with both thalidomide ${ }^{27,29,52,53,73}$ and bortezo$\mathrm{mib}^{52,53,69,70}$ has been associated with a substantially increased risk of peripheral neuropathy, which can be dose-limiting, while continuous and maintenance lenalidomide therapies have resulted in increased rates of hematologic toxicity, notably grade $3 / 4$ neutropenia $^{25,40,73}$, as well as chronic diarrhea ${ }^{91}$. Lenalidomide has also been associated with an increased risk of SPMs; in the meta-analysis of phase 3 studies of lenalidomide maintenance post ASCT, the cumulative rates of hematologic and solid SPMs prior to disease progression on lenalidomide maintenance were $5.3 \%$ and $5.8 \%$, vs. $0.8 \%$ and $2.0 \%$ with placebo/observation ${ }^{32}$. However, as the authors highlight, this risk is outweighed by the significantly reduced risk of disease progression with lenalidomide maintenance.

Limited QoL data have been reported from studies of long-term treatment approaches; however, the available findings appear promising, suggesting that such therapies do not typically have an adverse impact on QoL 
Table 7 QoL data reported from studies of long-term treatment approaches.

\begin{tabular}{|c|c|c|c|}
\hline Study & Treatment & QoL instruments & Key QoL findings \\
\hline Myeloma IX $X^{92}$ & $\begin{array}{l}\text { T ( } 50-100 \mathrm{mg} / \text { day to PD) vs. no maintenance } \\
\text { post ASCT }\end{array}$ & $\begin{array}{l}\text { EORTC QLQ-C30/QLQ- } \\
\text { MY24 }\end{array}$ & $\begin{array}{l}\text { - Minimal effects of thalidomide maintenance on } \\
\text { various subscales } \\
\text {-Small significant difference in favor of observation } \\
\text { only for Global Health Status/QoL at } 3 \text { months ( }-3.39 \text {, } \\
p=0.02) \\
\text { - No significant differences for Pain, Fatigue or Physical } \\
\text { Functioning } \\
\text {-Constipation worse with thalidomide maintenance vs. } \\
\text { observation at } 3 \text { and } 6 \text { months }\end{array}$ \\
\hline $\begin{array}{l}\text { NCIC-CTG Myeloma } \\
10^{28}\end{array}$ & $\begin{array}{l}\text { TP (T } 200 \mathrm{mg} / \mathrm{d}, \text { P } 50 \mathrm{mg} \text { Q2d; up to } 4 \text { years) vs. } \\
\text { observation post ASCT }\end{array}$ & $\begin{array}{l}\text { EORTC QLQ-C30 / trial- } \\
\text { specific disease module }\end{array}$ & $\begin{array}{l}\text {-QoL inferior with TP vs. observation for cognitive } \\
\text { function domain and for symptoms of dyspnea, } \\
\text { constipation, thirst, swelling in legs, numbness, dry } \\
\text { mouth, and balance problems, reflecting the toxicity } \\
\text { profile reported with this regimen } \\
\text {-QoL scores improved with TP vs. observation for } \\
\text { appetite and sleep }\end{array}$ \\
\hline FIRST $^{64,93,94}$ & $\begin{array}{l}\text { Continuous Rd vs. Rd ( } 18 \text { cycles) vs. MPT } \\
(72 \text { weeks) } \\
\text { QoL instruments were only administered at } \\
\text { specific time-points up to and including cycle 18, } \\
\text { plus at the end of the study. Thus, it was not } \\
\text { feasible to compare QoL between the continuous } \\
\text { Rd and Rd18 treatment arms, as treatment was } \\
\text { essentially the same through the QoL data } \\
\text { collection period }\end{array}$ & $\begin{array}{l}\text { EORTC QLQ-C30/QLQ- } \\
\text { MY20/EQ-5D }\end{array}$ & $\begin{array}{l}\text { - Consistent positive impact on patients' QoL with long- } \\
\text { term Rd, with improvements from baseline through } \\
18 \text { months reported across subscales with continuous } \\
\text { Rd/Rd18 } \\
\text {-Significant improvements from baseline in all arms for } \\
\text { Pain, Disease Symptoms, Global Health Status, Physical } \\
\text { Functioning, EQ-5D Health Utility, and Fatigue } \\
\text {-Rd showed clinically meaningful improvements in } \\
\text { Pain domain, vs. none with MPT } \\
\text {-Rd showed a significantly greater reduction in Disease } \\
\text { Symptoms vs. MPT at month } 3 \\
\text { - Treatment Side Effects domain worsened from } \\
\text { baseline in all arms, but scores were significantly better } \\
\text { with Rd vs. MPT } \\
\text {-Predicted QoL scores beyond } 18 \text { months of Rd } \\
\text { suggested that QoL improvements were maintained or } \\
\text { improved }\end{array}$ \\
\hline $\begin{array}{l}\text { Phase } 3 \text { LenaMain } \\
\text { study }\end{array}$ & $\begin{array}{l}\text { R } 25 \mathrm{mg} \text { vs. R } 5 \mathrm{mg} \text { (to PD) following } 6 \text { months of } \\
\text { post-ASCT consolidation }\end{array}$ & EORTC QLQ-C30 & $\begin{array}{l}\text { - No overall adverse impact on QoL subscales with } \\
\text { either maintenance dose } \\
\text { - Mean change in Global Health Status/QoL of }-4 \text { vs. }-8 \\
\text { after } 2 \text { years } \\
\text {-Trend for better overall QoL in the higher-dose arm, } \\
\text { including significantly better role functioning, but with } \\
\text { a significantly greater increase from baseline in } \\
\text { diarrhea symptom score } \\
\text {-Further illustrating the importance of evaluating } \\
\text { benefit/risk balance, the } 25 \mathrm{mg} \text { dose was associated } \\
\text { with significantly longer event-free survival but a } 10 \% \\
\text { increase in grade } 3 / 4 \text { infections per year }\end{array}$ \\
\hline $\begin{array}{l}\text { Connect }{ }^{\circledR} \mathrm{MM} \\
\text { registry }^{49}\end{array}$ & $\begin{array}{l}\mathrm{R} \text { maintenance vs any maintenance vs. no } \\
\text { maintenance }\end{array}$ & FACT-MM, EQ-5D, BPI & $\begin{array}{l}\text { - No adverse impact on QoL of lenalidomide or any } \\
\text { maintenance compared to no maintenance } \\
\text {-FACT-MM, EQ-5D, and BPI scores improved post ASCT } \\
\text { in all groups, with no significant differences in change } \\
\text { from baseline }\end{array}$ \\
\hline TOURMALINE-MM3 $3^{58}$ & $\begin{array}{l}\text { Ixazomib ( } 3-4 \mathrm{mg}, \mathrm{d} 1,8,15,28-\mathrm{d} \text { cycles; up to } 2 \\
\text { years) vs. placebo post ASCT }\end{array}$ & $\begin{array}{l}\text { EORTC QLQ-C30/QLQ- } \\
\text { MY20 }\end{array}$ & $\begin{array}{l}\text { - No detrimental impact on patients' QoL with ixazomib } \\
\text { compared to placebo } \\
\text {-Similar mean scores maintained from study entry to } \\
\text { end of treatment in both groups, including for } \\
\text { functioning, symptoms, and side-effects scales, except } \\
\text { Nausea or Vomiting and Diarrhea, which were } \\
\text { negatively affected in the ixazomib arm }\end{array}$ \\
\hline
\end{tabular}

ASCT autologous stem cell transplant, BPI Brief Pain Inventory, EORTC European Organisation for Research and Treatment of Cancer, EQ EuroQoL, FACT Functional Assessment of Cancer Therapy, MPT melphalan-prednisone-thalidomide, $P$ prednisone, $P D$ progressive disease, Q2d every other day, QLQ quality of life questionnaire, QoL quality of life, $R$ lenalidomide, $R d(18)$ lenalidomide-dexamethasone (for 18 cycles), $T$ thalidomide, $T P$ thalidomide-prednisone.

$($ Table 7) 28,49,58,64,92-95. However, commonly used QoL instruments may not capture all aspects of importance to patients receiving long-term therapy, e.g. sexual functioning. It is important to consider that some patients may not necessarily value a PFS benefit with long-term treatment approaches if not associated with better QoL or a treatment-free interval.
The impact on patients of the burden of prolonged treatment also requires consideration. This treatment burden may arise due to the need for repeated trips for hospital or physician appointments, or for repeated intravenous or subcutaneous drug administrations ${ }^{96}$. These inconveniences of receiving treatment may limit the feasibility of continuous or maintenance therapy with 
some agents in the real-world setting ${ }^{17}$. As reviewed herein, studies suggest that prolonged treatment is associated with improved PFS, and so novel approaches may be required to enable patients to continue therapy for as long as possible to achieve this PFS benefit; for example, switching from a parenterally administered to an oral therapy may make prolonged proteasome inhibitor therapy more feasible in the community setting, an approach currently being explored with ixazomib in the ongoing US MM-6 trial $^{97}$.

\section{Pharmacoeconomics of long-term treatment approaches}

While long-term treatment approaches are associated with improved outcomes, they may potentially be associated with a substantial economic impact due to patients receiving novel agents for a period of several years. Conversely, however, there may be long-term economic benefit as the efficacy of these approaches may delay the need for subsequent therapy and may reduce the healthcare burden due to disease-related side-effects. The economic impact/benefit may depend on the time period being evaluated and on whether the long-term treatment approach is associated only with a PFS benefit or also with an OS benefit. Thus, pharmacoeconomic evaluation is important to the emerging paradigm of continuous therapy. A number of analyses related to lenalidomide maintenance therapy have already been reported (Table $8)^{48,98-100}$.

\section{Conclusions}

The paradigm of long-term treatment is becoming increasingly well-established in different treatment settings in NDMM, with data reported on multiple agents and regimens in each setting clearly demonstrating the value of prolonged treatment duration on PFS and, in some cases (primarily lenalidomide as post-ASCT maintenance), OS. Ongoing studies are anticipated to provide further confirmation of this PFS and possibly OS benefit over the coming 5 years and may result in the addition of numerous novel therapy options to the long-term treatment armamentarium, providing physicians with a greater selection of therapeutic pathways for patients.

In order to optimize individual patient outcomes, it will be important to elucidate which continuous therapy and maintenance treatment approaches are most appropriate for which patient subgroups, taking into account not only clinical efficacy and safety but also tolerability, QoL, and patients' perspectives regarding the feasibility, convenience, and burden of long-term treatment. For example, some patients may prefer to maximize QoL and enjoy a treatment-free interval rather than have a PFS benefit with no QoL improvement with long-term therapy. To further inform such treatment decisions, longer follow-up

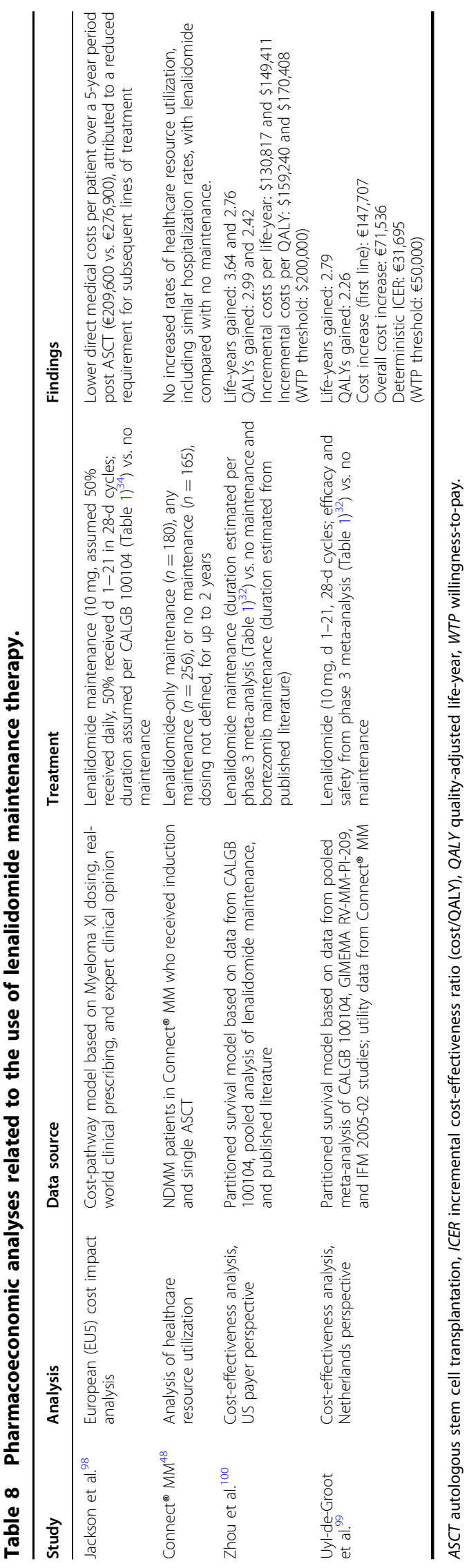


of ongoing studies will be important to determine whether OS benefits are seen with long-term treatment approaches other than lenalidomide as post-ASCT maintenance. Ongoing studies of continuous/maintenance therapy with monoclonal antibodies may hold particular promise in this regard. Additional randomized comparisons of different treatment durations and intensities, such as between more intense fixed-duration and continuous approaches, would also be of value for determining optimal treatment duration in different patients; such evidence-based information is not currently available.

Various other clinically important questions remain to be answered regarding continuous therapy and maintenance, such as optimal treatment duration, dosing schedule, the potential role of MRD evaluation in guiding decisions regarding continuation of treatment (and potentially as a regulatory endpoint), and how best to tailor treatment duration and intensity in the context of patient age and fitness, in order to provide optimal outcomes. The data in this review and the breadth of ongoing phase 3 studies offer encouragement that further improvements in patient survival will result from these long-term treatment approaches, potentially transforming MM into a chronic condition for many patients.

\section{Acknowledgements}

The authors acknowledge Steve Hill, Ph.D., of FireKite, an Ashfield company, part of UDG Healthcare plc, for professional medical writing support, which was funded by Millennium Pharmaceuticals Inc, Cambridge, MA, USA, a wholly owned subsidiary of Takeda Pharmaceutical Company Limited, and complied with Good Publication Practice-3 (GPP3) guidelines (Battisti, W. P. et al. Ann. Intern. Med. 163, 461-464 (2015)), and Renda Ferrari, Ph.D. (Millennium Pharmaceuticals, Inc.), for contributing to the editorial and scientific content of the manuscript.

\footnotetext{
Author details

'Hematology \& Medical Oncology, Department of Clinical Therapeutics, National and Kapodistrian University of Athens, School of Medicine, Athens, Greece. ${ }^{2}$ University of Chicago Medical Center, Chicago, IL, USA. ${ }^{3}$ Roswell Park Comprehensive Cancer Center, Buffalo, NY, USA. ${ }^{4}$ Department of Lymphoma/ Myeloma, The University of Texas MD Anderson Cancer Center, Houston, TX, USA. ${ }^{5}$ Hematology Department, University Hospital Purpan, Toulouse, France. ${ }^{6}$ Hematology Department, Hospital Clinic, Institut de Investigacions Biomediques August Pi I Sunyer (IDIBAPS), Barcelona, Spain. ${ }^{7}$ Department of Internal Medicine V, University Medical Hospital and National Center of Tumor Diseases, University of Heidelberg, Heidelberg, Germany. ${ }^{8}$ Department of Oncology, Hematology and Bone Marrow Transplantation with Section of Pneumology, University Medical Center Hamburg-Eppendorf, Hamburg, Germany. ${ }^{9}$ Oxford University Hospitals, NHS Foundation Trust, Oxford, UK. ${ }^{10}$ Department of Hematology, Cancer Center Amsterdam, Amsterdam University Medical Center, VU University Amsterdam, Amsterdam, The Netherlands. "Malignant Haematology and Stem Cell Transplantation Service, Alfred Health-Monash University, Melbourne, Australia. ${ }^{12}$ National Taiwan University, Taipei, Taiwan. ${ }^{13}$ Department of Hematology, Peking University People's Hospital and Peking University Institute of Hematology, Beijing, China. ${ }^{14}$ Department of Hematology, National Hospital Organization Okayama Medical Center, Okayama, Japan. ${ }^{15}$ Department of Hematology and Oncology, Nagoya City University Graduate School of Medical Sciences, Nagoya, Japan. ${ }^{16}$ Department of Haematology-Oncology, National University Cancer Institute, National University Health System, and Cancer Science Institute of Singapore, National University of Singapore, Singapore, Singapore. ${ }^{17}$ Department of Internal Medicine, University of Nebraska Medical Center, Omaha, NE, USA.
}

${ }^{18}$ Department of Haematology, Manchester University Hospitals NHS Foundation Trust, Manchester, UK. ${ }^{19}$ Faculty of Biology, Medicine and Health, School of Medical Science, Division of Cancer Science, University of Manchester, Manchester, UK. ${ }^{20}$ Millennium Pharmaceuticals, Inc., a wholly owned subsidiary of Takeda Pharmaceutical Company Limited, Cambridge, MA, USA. ${ }^{21}$ Dana-Farber Cancer Institute, Boston, MA, USA

\section{Conflict of interest}

M.A.D.: Genesis Pharma: Research Funding; Novartis: Consultancy, Honoraria; Amgen Inc, Celgene Corporation, Janssen Biotech Inc, Onyx Pharmaceuticals, an Amgen subsidiary, Takeda Oncology: Consultancy, Honoraria, Other: Advisory Committee. A.J.J.: Consultant and Advisory Boards with honoraria for AbbVie, Amgen, BMS, Celgene, Janssen, Juno, Karyopharm, SkylineDx, Takeda. P.L.M.: honoraria from Celgene, Bristol-Myers Squibb, Sanofi, Takeda, The Binding Site, and Karyopharm Therapeutics; consulting or advisory role for Celgene, Janssen, Bristol-Myers Squibb, Sanofi, and Karyopharm Therapeutics; research funding from Celgene. RZO: research funding from BioTheryX; honoraria from Amgen, Bristol-Myers Squibb, Celgene Corporation, Janssen, Millennium Pharmaceuticals, and Onyx Pharmaceuticals; member of advisory boards for Amgen, Bristol-Myers Squibb, Celgene Corporation, Incyte, Juno, Kite, Legend Biotech, Sanofi-Aventis, and Takeda Pharmaceuticals. M.A.: None. J.B.: honoraria from Janssen, Celgene, Amgen, and Takeda; grant support from Janssen and Celgene. H.G.: advisory boards for Adaptive Biotechnology, Amgen, Bristol-Myers Squibb, Celgene, Janssen, Sanofi, and Takeda; research funding from Amgen, Bristol-Myers Squibb, Celgene, Chugai, Janssen, Sanofi, Mundipharma, Takeda, and Novartis; honoraria from ArtTempi, Bristol-Myers Squibb, Celgene, Chugai, Janssen, and Novartis. K.C.W.: Advisory boards for Amgen, Bristol-Myers Squibb, Adaptive Biotech, Celgene, Janssen, Juno, Takeda, Sanofi; honoraria from Amgen, Bristol-Myers Squibb, Celgene, Janssen, and Takeda; research funding from Amgen, Celgene, Janssen, and Sanofi. K.R. Takeda: honoraria, research funding; Amgen: honoraria; Janssen: honoraria; Celgene: honoraria, research funding; Adaptive Biotech: Honoraria; Oncopeptides: Honoraria. S.Z: research funding from and advisory boards for Takeda, Celgene, and Janssen. A.S.: consultancy for Specialised Therapeutics Australia; honoraria from Takeda, Celgene, Janssen, and Amgen; speaker bureaus for Takeda, Celgene, and Janssen; research funding from Takeda, Celgene, Janssen, and GlaxoSmithKline. J.S.Y.H.: honoraria and advisory boards for Takeda, Celgene, and Janssen. J.L.: None. K.S.: research funding from Takeda, MSD, Celgene, GSK, Novartis, Ono, Janssen, Abbvie, Sanofi, Bristol-Myers Squibb, Alexion-pharma, Diichi-Sankyo; honoraria from Takeda, Celgene, Ono, Bristol-Myers Squibb. S.I.: research funding from Takeda, Ono, Janssen, Celgene, Novartis, Chugai, Abbvie, Bristol-Myers Squibb, Kyowa-Hakko Kirin, Merck Sharp \& Dohme, Daiichi Sankyo, Gilead; honoraria from Takeda, Janssen, Celgene, Ono, Daiichi Sankyo, and Bristol-Myers Squibb. W.-J.C.: honoraria from Takeda. S.A.H.: Consultant: Celgene, Sorrento, GSK; Advisory boards: Adaptive Biotechnologies, Takeda. A.R.: Consultancy for Sanofi and Takeda; honoraria from Janssen, Takeda, Celgene, Novartis, Amgen; Advisory board for Novartis, Janssen, Amgen. T.S., R.L., A.P.: Employment, Millennium Pharmaceuticals, Inc., a wholly owned subsidiary of Takeda Pharmaceutical Company Limited. T.S.: also affiliated with Department of Hematology, Charles University General Hospital, Prague, Czech Republic. K.C.A.: Membership on an entity's Board of Directors or advisory committees for Bristol-Myers Squibb, Gilead Sciences, Celgene, Janssen, Sanofi-Aventis, Millennium Pharmaceuticals; Scientific founder: Oncopep, C4 Therapeutics.

\section{Publisher's note}

Springer Nature remains neutral with regard to jurisdictional claims in published maps and institutional affiliations.

Received: 13 May 2019 Revised: 13 September 2019 Accepted: 18 September 2019

Published online: 13 February 2020

\section{References}

1. Gay, F. et al. From transplant to novel cellular therapies in multiple myeloma: European Myeloma Network guidelines and future perspectives. Haematologica 103, 197-211 (2018). 
2. Ludwig, H. \& Zojer, N. Fixed duration vs continuous therapy in multiple myeloma. Hematology Am. Soc. Hematol. Educ. Program 2017, 212-222 (2017).

3. Palumbo, A. et al. Continuous therapy versus fixed duration of therapy in patients with newly diagnosed multiple myeloma. J. Clin. Oncol. 33, 3459-3466 (2015).

4. Facon, T. et al. Final analysis of survival outcomes in the phase 3 FIRST trial of up-front treatment for multiple myeloma. Blood 131, 301-310 (2018).

5. Pulte, E. D. et al. FDA approval summary: lenalidomide as maintenance therapy after autologous stem cell transplant in newly diagnosed multiple myeloma. Oncologist 23, 734-739 (2018).

6. Kumar, S. K. et al. NCCN guidelines insights: multiple myeloma, version 3.2018. J. Natl. Compr. Canc. Netw. 16, 11-20 (2018).

7. Gonsalves, W. I. et al. Utilization of hematopoietic stem cell transplantation for the treatment of multiple myeloma: a Mayo Stratification of Myeloma and Risk-Adapted Therapy (mSMART) consensus statement. Bone Marrow Transplant. 54, 353-367 (2019).

8. Palumbo, A. et al. International Myeloma Working Group consensus statement for the management, treatment, and supportive care of patients with myeloma not eligible for standard autologous stem-cell transplantation. J. Clin. Oncol. 32, 587-600 (2014).

9. Sonneveld, P. et al. Treatment of multiple myeloma with high-risk cytogenetics: a consensus of the International Myeloma Working Group. Blood 127 2955-2962 (2016).

10. Moreau, P. et al. Multiple myeloma: ESMO Clinical Practice Guidelines for diagnosis, treatment and follow-up. Ann. Oncol. 28(suppl_4), iv52-iv61 (2017).

11. Kantar Health. Treatment Architecture: United States, Multiple Myeloma CancerMPact ${ }^{\circledR}$ (Kantar Health, United States, 2017).

12. Raab, M. S. et al. Multiple myeloma: practice patterns across Europe. Br. J. Haematol. 175, 66-76 (2016).

13. Anderson, K. C. et al. The role of minimal residual disease testing in myeloma treatment selection and drug development: current value and future applications. Clin. Cancer Res. 23, 3980-3993 (2017).

14. Wilke, T. et al. Treatment of relapsed refractory multiple myeloma: which new PI-based combination treatments do patients prefer? Patient Prefer. Adherence 12, 2387-2396 (2018).

15. Romanus, D. et al. Treatment satisfaction and burden of illness with oral vs injectable multiple myeloma therapy in patients with newly diagnosed disease (NDMM). Value Health 20, A454 (abstract) (2017).

16. Merola, D., Yong, C., Noga, S. J. \& Shermock, K. M. Costs associated with productivity loss among U.S. patients newly diagnosed with multiple myeloma receiving oral versus injectable chemotherapy. J. Manag. Care Spec. Pharm. 24, 1019-1026 (2018).

17. Chng, W. J. et al. Addressing unmet medical needs in maintenance treatment for newly diagnosed multiple myeloma (NDMM): current treatment landscape and emerging therapeutic options. Hemasphere 2(S1), 1001 (abstract PB2252) (2018).

18. Morgan, G. J., Walker, B. A. \& Davies, F. E. The genetic architecture of multiple myeloma. Nat. Rev. Cancer 12, 335-348 (2012).

19. Lahuerta, J. J. et al. Depth of response in multiple myeloma: a pooled analysis of three PETHEMAVGEM clinical trials. J. Clin. Oncol. 35, 2900-2910 (2017).

20. Lehners, N. et al. Analysis of long-term survival in multiple myeloma after first-line autologous stem cell transplantation: impact of clinical risk factors and sustained response. Cancer Med. 7, 307-316 (2018).

21. Fernández, R. A. et al. Maintenance treatment with lenalidomide for multiple myeloma increases the proportion of MRD negative (Flow-/PET-CT-) patients. Blood 130(Suppl 1), 3098 (abstract) (2017).

22. Mateos, M. V. et al. Daratumumab plus bortezomib, melphalan, and prednisone for untreated myeloma. N. Engl. J. Med. 378, 518-528 (2018).

23. Mateos, M. V. et al. GEM2005 trial update comparing VMPNTP as induction in elderly multiple myeloma patients: do we still need alkylators? Blood $\mathbf{1 2 4}$ 1887-1893 (2014).

24. Gambella, M. et al. Minimal residual disease by flow cytometry and allelicspecific oligonucleotide real-time quantitative polymerase chain reaction in patients with myeloma receiving lenalidomide maintenance: a pooled analysis. Cancer 125, 750-760 (2019).

25. Jackson, G. et al. Lenalidomide maintenance significantly improves outcomes compared to observation irrespective of cytogenetic risk: results of the Myeloma XI Trial. Blood 130(Suppl 1), 436 (abstract) (2017).
26. Morgan, G. J. et al. The role of maintenance thalidomide therapy in multiple myeloma: MRC Myeloma IX results and meta-analysis. Blood 119, 7-15 (2012).

27. Lokhorst, H. M. et al. A randomized phase 3 study on the effect of thalidomide combined with adriamycin, dexamethasone, and high-dose melphalan, followed by thalidomide maintenance in patients with multiple myeloma. Blood 115, 1113-1120 (2010).

28. Stewart, A. K. et al. A randomized phase 3 trial of thalidomide and prednisone as maintenance therapy after ASCT in patients with MM with a quality-of-life assessment: the National Cancer Institute of Canada Clinicals Trials Group Myeloma 10 Trial. Blood 121, 1517-1523 (2013).

29. van de Donk, N. W. et al. Thalidomide before and after autologous stem cell transplantation in recently diagnosed multiple myeloma (HOVON-50): longterm results from the phase 3, randomised controlled trial. Lancet Haematol. 5, e479-e492 (2018)

30. Ludwig, $\mathrm{H}$. et al. IMWG consensus on maintenance therapy in multiple myeloma. Blood 119, 3003-3015 (2012).

31. Morgan, G. J. et al. Long-term follow-up of MRC Myeloma IX trial: Survival outcomes with bisphosphonate and thalidomide treatment. Clin. Cancer Res. 19, 6030-6038 (2013).

32. McCarthy, P. L. et al. Lenalidomide maintenance after autologous stem-cell transplantation in newly diagnosed multiple myeloma: a meta-analysis. J. Clin. Oncol. 35, 3279-3289 (2017).

33. Holstein, S. A. et al. Updated analysis of CALGB (Alliance) 100104 assessing lenalidomide versus placebo maintenance after single autologous stem-cell transplantation for multiple myeloma: a randomised, double-blind, phase 3 trial. Lancet Haematol. 4, e431-e442 (2017).

34. McCarthy, P. L. et al. Lenalidomide after stem-cell transplantation for multiple myeloma. N. Engl. J. Med. 366, 1770-1781 (2012).

35. McCarthy, P. L. et al. Survival analysis from the CALGB study of lenalidomide maintenance therapy in newly diagnosed multiple myeloma postautologous stem cell transplantation adjusted for crossover (Alliance 100104). Blood 132(Suppl 1), 4737 (abstract) (2018).

36. Attal, M. et al. Lenalidomide maintenance after stem-cell transplantation for multiple myeloma. N. Engl. J. Med. 366, 1782-1791 (2012).

37. Palumbo, A. et al. Autologous transplantation and maintenance therapy in multiple myeloma. N. Engl. J. Med. 371, 895-905 (2014).

38. Cherniawsky, H. et al. Lenalidomide maintenance does not negatively impact overall and progression free survival using lenalidomide-based regimens for multiple myeloma in first relapse. Blood 132(Suppl 1), 5643 (abstract) (2018).

39. Jagannath, S. et al. Treatment choices and outcomes for patients with multiple myeloma after relapse on lenalidomide maintenance therapy: results from the Connect MM Registry. Blood 132(Suppl 1), 3232 (abstract) (2018).

40. Jackson, G. H. et al. Lenalidomide maintenance versus observation for patients with newly diagnosed multiple myeloma (Myeloma XI): a multicentre, open-label, randomised, phase 3 trial. Lancet Oncol. 20, 57-73 (2019).

41. Gay, F. et al. Chemotherapy plus lenalidomide versus autologous transplantation, followed by lenalidomide plus prednisone versus lenalidomide maintenance, in patients with multiple myeloma: a randomised, multicentre, phase 3 trial. Lancet Oncol. 16, 1617-1629 (2015).

42. Attal, M. et al. Lenalidomide, bortezomib, and dexamethasone with transplantation for myeloma. N. Engl. J. Med. 376, 1311-1320 (2017).

43. Thomas, S. K. et al. Updated results of a phase II study of lenalidomideelotuzumab as maintenance therapy post-autologous stem cell transplant (AuSCT) in patients (Pts) with multiple myeloma (MM). Blood 132(Suppl 1), 1982 (abstract) (2018).

44. de Tute, R. M. et al. Minimal residual disease in the maintenance setting in myeloma: prognostic significance and impact of lenalidomide. Blood $\mathbf{1 3 0}$ (Suppl 1), 904 (abstract) (2017).

45. Dimopoulos, M. A. et al. All-oral ixazomib, cyclophosphamide, and dexamethasone for transplant-ineligible patients with newly diagnosed multiple myeloma. Eur. J. Cancer 106, 89-98 (2019).

46. Oliva, S. et al. Minimal residual disease (MRD) by multiparameter flow cytometry (MFC) in transplant eligible patients with newly diagnosed multiple myeloma (MM): results from the EMN02/HO95 phase 3 trial. Haematologica 102(s2), 2 (abstract S102) (2017).

47. Jagannath, S. et al. Impact of post-ASCT maintenance therapy on outcomes in patients with newly diagnosed multiple myeloma in Connect MM. Blood Adv. 2, 1608-1615 (2018). 
48. Rifkin, R. M. et al. Treatment outcomes and health care resource utilization in patients with newly diagnosed multiple myeloma receiving lenalidomideonly maintenance, any maintenance, or no maintenance: results from the Connect MM Registry. Clin. Ther. 40, 1193-1202 e1191 (2018).

49. Abonour, R. et al. Impact of post-transplantation maintenance therapy on health-related quality of life in patients with multiple myeloma: data from the Connect(R) MM Registry. Ann. Hematol. 97, 2425-2436 (2018).

50. Chakraborty, R. et al. Outcomes of maintenance therapy with lenalidomide or bortezomib in multiple myeloma in the setting of early autologous stem cell transplantation. Leukemia 32, 712-718 (2018)

51. Yang, $C$. et al. Tolerability and efficacy of post transplant lenalidomide maintenance therapy in multiple myeloma: a real world single centre experience. Blood 130(Suppl 1), 3462 (abstract) (2017).

52. Goldschmidt, $H$. et al. Bortezomib before and after high-dose therapy in myeloma: long-term results from the phase III HOVON-65/GMMG-HD4 trial. Leukemia 32, 383-390 (2018).

53. Sonneveld, P. et al. Bortezomib induction and maintenance treatment in patients with newly diagnosed multiple myeloma: results of the randomized phase III HOVON-65/ GMMG-HD4 trial. J. Clin. Oncol. 30, 2946-2955 (2012).

54. Rosinol, L. et al. Bortezomib and thalidomide maintenance after stem cell transplantation for multiple myeloma: a PETHEMA/GEM trial. Leukemia $\mathbf{3 1}$ 1922-1927 (2017).

55. Merz, M. et al. Prognostic significance of cytogenetic heterogeneity in patients with newly diagnosed multiple myeloma. Blood Adv. 2, 1-9 (2018).

56. Nooka, A. K. et al. Consolidation and maintenance therapy with lenalidomide, bortezomib and dexamethasone (RVD) in high-risk myeloma patients. Leukemia 28, 690-693 (2014).

57. Nadiminti, K. et al. A single autologous stem cell transplant (ASCT) followed by two years of post-transplant therapy in recently diagnosed elderly multiple myeloma ( $\mathrm{MM}$ ) patients. safety and response results from the prospective phase II trial (NCT01849783). Blood 132(Suppl 1), 2153 (abstract) (2018).

58. Dimopoulos, M. A. et al. Oral ixazomib maintenance following autologous stem cell transplantation (TOURMALINE-MM3): a double-blind, randomised, placebo-controlled phase 3 trial. Lancet 393, 253-264 (2018).

59. Patel, K. K. et al. Update on a phase II study of ixazomib with lenalidomide as maintenance therapy following autologous stem cell transplant in patients with multiple myeloma. Blood 130(Suppl 1), 437 (abstract) (2017).

60. Goldschmidt, H. et al. Response-adapted lenalidomide maintenance in newly diagnosed, transplant-eligible multiple myeloma: results from the multicenter phase III GMMG-MM5 trial. Blood 130(Suppl 1), 400 (abstract) (2017).

61. Cerrato, C. et al. Maintenance in myeloma patients achieving complete response after upfront therapy: a pooled analysis. J. Cancer Res. Clin. Oncol. 144, 1357-1366 (2018).

62. Solovev, M. V. et al. Efficacy of maintenance therapy following auto-HSCT depending on MRD status in patients with multiple myeloma. Blood 132 (Suppl 1), 3432 (abstract) (2018).

63. Gay, F. et al. Carfilzomib-lenalidomide-dexamethasone (KRd) inductionautologous transplant (ASCT)-Krd consolidation vs KRd 12 cycles vs carfilzomib-cyclophosphamide-dexamethasone (KCd) induction-ASCT-KCd consolidation: analysis of the randomized forte trial in newly diagnosed multiple myeloma (NDMM). Blood 132(Suppl 1), 121 (abstract) (2018).

64. Benboubker, L. et al. Lenalidomide and dexamethasone in transplantineligible patients with myeloma. N. Engl. J. Med. 371, 906-917 (2014).

65. Bahlis, N. J. et al. Benefit of continuous treatment for responders with newly diagnosed multiple myeloma in the randomized FIRST trial. Leukemia $\mathbf{3 1}$ 2435-2442 (2017).

66. Dimopoulos, M. A. et al. Impact of renal impairment on outcomes with lenalidomide and dexamethasone treatment in the FIRST trial, a randomized, open-label phase 3 trial in transplant-ineligible patients with multiple myeloma. Haematologica 101, 363-370 (2016).

67. Larocca, A. et al. Efficacy and feasibility of dose/schedule-adjusted Rd-R vs. continuous $\mathrm{Rd}$ in elderly and intermediate-fit newly diagnosed multiple myeloma (NDMM) patients: RV-MM-PI-0752 phase III randomized study. Blood 132(Suppl 1), 305 (abstract) (2018).

68. Facon, T. et al. Daratumumab plus lenalidomide and dexamethasone for untreated myeloma. N. Engl. J. Med. 380, 2104-2115 (2019).

69. Durie, B. G. et al. Bortezomib with lenalidomide and dexamethasone versus lenalidomide and dexamethasone alone in patients with newly diagnosed myeloma without intent for immediate autologous stem-cell transplant
(SWOG S0777): a randomised, open-label, phase 3 trial. Lancet 389, 519-527 (2017).

70. Durie, B. G. M. et al. Longer term follow up of the a randomized phase III trial SWOG S0777: bortezomib, lenalidomide and dexamethasone vs. lenalidomide and dexamethasone in patients (Pts) with previously untreated multiple myeloma without an intent for immediate autologous stem cell transplant (ASCT). Blood 132(Suppl 1), 1992 (abstract) (2018).

71. Ramasamy, K., Thom, H., D'Souza, V. K., Buchanan, V. \& Dhanasiri, S. Relative efficacy of treatment options in newly diagnosed multiple myeloma: results from a systematic literature review and network meta-analysis. Blood 132 (Suppl 1), 4744 (abstract) (2018)

72. Palumbo, A. et al. Continuous lenalidomide treatment for newly diagnosed multiple myeloma. N. Engl. J. Med. 366, 1759-1769 (2012).

73. Zweegman, S. et al. Melphalan, prednisone, and lenalidomide versus melphalan, prednisone, and thalidomide in untreated multiple myeloma. Blood 127, 1109-1116 (2016).

74. Stewart, A. K. et al. Melphalan, prednisone, and thalidomide vs melphalan, prednisone, and lenalidomide (ECOG E1A06) in untreated multiple myeloma. Blood 126, 1294-1301 (2015).

75. Magarotto, V. et al. Triplet vs doublet lenalidomide-containing regimens for the treatment of elderly patients with newly diagnosed multiple myeloma. Blood 127, 1102-1108 (2016).

76. Mateos, M. V. et al. Maintenance therapy with bortezomib plus thalidomide or bortezomib plus prednisone in elderly multiple myeloma patients included in the GEM2005MAS65 trial. Blood 120, 2581-2588 (2012).

77. Palumbo, A. et al. Bortezomib-melphalan-prednisone-thalidomide followed by maintenance with bortezomib-thalidomide compared with bortezomibmelphalan-prednisone for initial treatment of multiple myeloma: a randomized controlled trial. J. Clin. Oncol. 28, 5101-5109 (2010).

78. Niesvizky, R. et al. Community-based phase IIIB trial of three UPFRONT bortezomib-based myeloma regimens. J. Clin. Oncol. 33, 3921-3929 (2015).

79. Bringhen, $\mathrm{S}$. et al. Phase $1 / 2$ study of weekly carfilzomib, cyclophosphamide, dexamethasone in newly diagnosed transplant-ineligible myeloma. Leukemia 32, 979-985 (2018).

80. Kumar, S. K. et al. Safety and tolerability of ixazomib, an oral proteasome inhibitor, in combination with lenalidomide and dexamethasone in patients with previously untreated multiple myeloma: an open-label phase 1/2 study. Lancet Oncol. 15, 1503-1512 (2014).

81. Richardson, P. G. et al. Twice-weekly ixazomib in combination with lenalidomide-dexamethasone in patients with newly diagnosed multiple myeloma. Br. J. Haematol. 182, 231-244 (2018).

82. San-Miguel, J. F. et al. A phase $\mathbf{l} / /$ dose-escalation study investigating all-oral ixazomib-melphalan-prednisone induction followed by single-agent ixazomib maintenance in transplant-ineligible newly diagnosed multiple myeloma. Haematologica 103, 1518-1526 (2018).

83. Kumar, S. K. et al. Ixazomib, lenalidomide, and dexamethasone in patients with newly diagnosed multiple myeloma: long-term follow-up including ixazomib maintenance. Leukemia 33, 1736-1746 (2019).

84. Dimopoulos, M. A. et al. Ixazomib maintenance therapy in newly diagnosed multiple myeloma: An integrated analysis of four phase $1 / \|$ studies. Eur $J$ Haematol 102, 494-503 (2019)

85. Stege, C. A. M. et al. Efficacy and tolerability of ixazomib, daratumumab and low dose dexamethasone (IDd) in unfit and frail newly diagnosed multiple myeloma (NDMM) patients; first interim safety analysis of the phase ॥ HOVON 143 study. Blood 132(Suppl 1), 596 (abstract) (2018).

86. Zweegman, S. et al. Ixazomib-thalidomide-low dose dexamethasone (ITd) induction followed by maintenance therapy with ixazomib or placebo in newly diagnosed multiple myeloma patients not eligible for autologous stem cell transplantation; results from the randomized phase II HOVON-126/ Nmsg 21\#13 trial. Blood 132(Suppl 1), 800 (abstract) (2018).

87. Dimopoulos, M. A. et al. One-year update of a phase 3 randomized study of daratumumab plus bortezomib, melphalan, and prednisone (DVMP) versus bortezomib, melphalan, and prednisone (VMP) in patients (Pts) with transplant-ineligible newly diagnosed multiple myeloma (NDMM): Alcyone. Blood 132(Suppl 1), 156 (abstract) (2018).

88. San-Miguel, J. F. et al. Treatment regimens for patients with newly diagnosed multiple myeloma who are ineligible for stem cell transplantation: a systematic literature review and network meta-analysis. Blood 132(Suppl 1), 4741 (abstract) (2018).

89. Dimopoulos, M. A. et al. A matching-adjusted indirect treatment comparison of daratumumab-bortezomib-melphalan-prednisone versus lenalidomide- 
dexamethasone continuous, lenalidomide-dexamethasone 18 months, and melphalan-prednisone-thalidomide. Blood 132(Suppl 1), 3551 (abstract) (2018).

90. Huang, J. et al. Lenalidomide vs bortezomib maintenance choice postautologous hematopoietic cell transplantation for multiple myeloma. Bone Marrow Transplant. 53, 701-707 (2018).

91. Ludwig, H. et al. Prevention and management of adverse events of novel agents in multiple myeloma: a consensus of the European Myeloma Network. Leukemia 32, 1542-1560 (2018).

92. Royle, K. L. et al. Quality of life during and following sequential treatment of previously untreated patients with multiple myeloma: findings of the Medical Research Council Myeloma IX randomised study. Br. J. Haematol. 182, 816-829 (2018)

93. Delforge, M. et al. Health-related quality-of-life in patients with newly diagnosed multiple myeloma in the FIRST trial: lenalidomide plus low-dose dexamethasone versus melphalan, prednisone, thalidomide. Haematologica 100, 826-833 (2015)

94. Vogl, D. T. et al. Long-term health-related quality of life in transplantineligible patients with newly diagnosed multiple myeloma receiving lenalidomide and dexamethasone. Leuk. Lymphoma 59, 398-405 (2018).

95. Boquoi, A. et al. Similar quality of life with $5 \mathrm{mg}$ versus $25 \mathrm{mg}$ lenalidomide maintenance after first-line high-dose therapy and autologous blood stem cell transplantation for multiple myeloma: results of the Lenamain trial. Blood 132(Suppl 1), 2003 (abstract) (2018).

96. Baz, R. et al. Development of a conceptual model to illustrate the impact of multiple myeloma and its treatment on health-related quality of life. Support Care Cancer 23, 2789-2797 (2015)

97. Rifkin, R. M. et al. Tourmaline US-MM6, an open-label, single-arm, multicenter study evaluating the effectiveness and safety of ixazomib in combination with lenalidomide and dexamethasone (IRd) in patients (pts) with newly diagnosed multiple myeloma (NDMM) switching from a bortezomib-based triplet induction regimen. Blood 130(Suppl 1), 5407 (abstract) (2017).

98. Jackson, G., Dutton, R., Zamagni, E., Hughes, R. \& Dhanasiri, S. Lenalidomide maintenance therapy post-autologous stem cell transplant: a healthcare cost-impact analysis in Europe. Blood 130(Suppl 1), 3405 (abstract) (2017).

99. Uyl-de Groot, C. A., Ramsden, R., Boersma, J, Zweegman, S. \& Dhanasiri, S. Lenalidomide as maintenance treatment for patients with newly diagnosed multiple myeloma post-autologous stem cell transplantation: a pharmacoeconomic assessment in the Netherlands. Blood 132(Suppl 1), 3555 (abstract) (2018).

100. Zhou, Z.-Y. et al. Cost-effectiveness analysis of lenalidomide for maintenance therapy after autologous stem cell transplant (ASCT) in newly diagnosed multiple myeloma (NDMM) patients: a United States payer perspective. Blood 132(Suppl 1), 3535 (abstract) (2018). 